\title{
A Two-Scale Approach for the Analysis of Propagating Three-Dimensional Fractures
}

\author{
J.P.A. Pereira ${ }^{a}$, D.-J. Kim ${ }^{b}$ and C.A. Duarte ${ }^{a, 1}$ \\ ${ }^{a}$ Department of Civil and Environmental Engineering, University of Illinois at Urbana-Champaign \\ 2122 Newmark Laboratory, 205 North Mathews Avenue, Urbana, Illinois 61801, USA \\ ${ }^{b}$ Department of Architectural Engineering, Kyung Hee University \\ Engineering Building, 1 Sochon-Dong Kihung-Gu, Yongin, Kyunggi-Do, Korea 446-701
}

\begin{abstract}
This paper presents a generalized finite element method (GFEM) for crack growth simulations based on a two-scale decomposition of the solution - a smooth coarse-scale component and a singular fine-scale component. The smooth component is approximated by discretizations defined on coarse finite element meshes. The fine-scale component is approximated by the solution of local problems defined in neighborhoods of cracks. Boundary conditions for the local problems are provided by the available solution at a crack growth step. The methodology enables accurate modeling of 3-D propagating cracks on meshes with elements that are orders of magnitude larger than those required by the FEM. The coarse-scale mesh remains unchanged during the simulation. This, combined with the hierarchical nature of GFEM shape functions, allows the recycling of the factorization of the global stiffness matrix during a crack growth simulation. Numerical examples demonstrating the approximating properties of the proposed enrichment functions and the computational performance of the methodology are presented.
\end{abstract}

KEY WORDS: Generalized FEM; Extended FEM; Fracture; Crack growth; Fatigue; Multi-scale; Globallocal analysis.

\section{Introduction}

The prediction of growth rate, shape, and trajectory of cracks in structural components is of great importance in several engineering applications. Relevant examples are the prediction of fatigue life of engine components and structural members. Realistic crack growth simulations require many crack propagation steps, several initial crack configurations, and, often, a non-linear analysis. As a result, the computing power required to solve this class of problems using existing methodologies can be formidable. Representative methods for three-dimensional crack growth simulations include the standard finite element method (FEM) with remeshing [60], the boundary element method (BEM) [36, 11], and the extended [38, 25, 57, 1, 8, 58] or generalized FEM $[18,48]$. These methods require the solution of the problem from scratch at each step of a crack growth simulation. As a result, each crack propagation step may take several hours even on teraflop computers [60].

\footnotetext{
${ }^{1}$ Correspondence to C.A. Duarte, Department of Civil and Environmental Engineering, University of Illinois at UrbanaChampaign, 2122 Newmark Laboratory, 205 North Mathews Avenue, Urbana, Illinois 61801, USA. Tel.: +1-217-244-2830; Fax: +1-217-265-8040. e-mail: caduarte@illinois.edu.
} 
This paper presents a generalized FEM for crack growth simulations that combines the concept of globallocal enrichments introduced in $[19,13]$ with the $h p$-GFEM for 3-D propagating fractures presented in [48]. The GFEM with global-local enrichments $\left(\right.$ GFEM $\left.^{\mathrm{gl}}\right)$ uses a two-scale decomposition of the solution of a fracture mechanics problem - a smooth coarse-scale component and a singular fine-scale component. The smooth component is approximated by a global (structural-scale) discretization defined on a coarse finite element mesh. The fine-scale (near crack) component is approximated by the solution of boundary value problems defined in neighborhoods of cracks. The partition of unity method $[4,3,34,14,15,16,12,40]$ is then used to create conforming global spaces enriched with the fine-scale problem solutions. A key point in this class of methods is the boundary conditions used in the fine-scale problems. In the GFEM ${ }^{\mathrm{gl}}$ presented here, the solution at a simulation step is used as boundary conditions for the fine-scale problems. The solutions of these problems, in turn, are used to define GFEM solution spaces at the next simulation step. A key idea introduced in this paper is to utilize as much as possible information available at a crack simulation step in order to reduce computational cost at the next step. The proposed GFEM ${ }^{\mathrm{gl}}$ takes advantage of the fact that crack increments, in all crack propagation algorithms we are aware, must be small for accurate predictions of crack paths in mixed-mode 3-D simulations. Thus, the solution away from the crack front does not change significantly between crack propagation steps.

The proposed methodology can be combined with the standard FEM while enabling accurate modeling of 3-D propagating cracks on meshes with elements that are orders of magnitude larger than those required by the FEM. Furthermore, only a few degrees of freedom are hierarchically added to the uncracked coarse-scale discretization regardless of the number of degrees of freedom required to solve the fine-scale problems. This enables the factorized matrix of the global problem to be recycled during a crack growth simulation. The numerical experiments presented in Section 4 show that the accuracy of the proposed GFEM ${ }^{\text {gl }}$ for crack growth is comparable to the $h p$-GFEM presented in [48] while being significantly more computationally efficient than available methods for this class of problems.

In this paper, we focus on crack growth problems modeled with the linear elastic fracture mechanics theory. However, the methodology presented here is not limited to this application. Several other classes of problems, like time-dependent ones and those involving non-linearities, are solved using a multi-step algorithm. Thus, the idea of using available information at a solution step to build approximation spaces for the next step is also applicable to them. This is demonstrated in [42] for time-dependent problems exhibiting sharp thermal gradients and in [29] for problems with localized material non-linearities. This broad applicability of the GFEM $^{\mathrm{gl}}$ is in contrast with other recently proposed multi-scale methods which rely on, e.g., analytically derived boundary conditions for fine-scale problems $[55,56]$. The GFEM $^{\mathrm{gl}}$ is also related to upscaling techniques proposed by $\mathrm{Hou}$ and $\mathrm{Xu}$ [27]. However, the solution spaces in the $\mathrm{GFEM}^{\mathrm{gl}}$ are conforming, while some of the methods presented in [27] are not.

Early methods for the analysis of propagating fractures based on multi-scale concepts include the work of Rashid on the arbitrary local mesh replacement method [51], and several works on the so-called S-method $[22,32]$. More recent methods aimed at crack growth modeling and based on multi-scale concepts include the multigrid methods proposed in [50,45]; the method of Guidault et al. [26] based on the LATIN method and domain decomposition concepts; the method of Pierres et al. [49] based on the LATIN method and augmented Lagrangian methods; the method of Ben Dhia and Jamond [7] which combines the extended FEM (XFEM) with the Arlequin method; the method of Galland et al. [24] based on global model reduction. A recent version of the s-method aimed at multi-scale failure simulations, is the reduced order s-method (rs-method) of Fish et al. [21, 43]. A related method aimed at modeling interactions among multiple static cracks is the multiscale method of Loehnert and Belytschko [33]. Other related methods for two-dimensional static cracks include the spider-XFEM [9] and the reduced basis enrichment for the XFEM [10] of Chahine et al.; the method of Menk and Bordas for fracture of bi-material systems [35]; the harmonic enrichment functions of Mousavi et al. [39] for two-dimensional branched cracks.

The outline of the paper is as follows. Section 2 briefly reviews the definition of approximation spaces used 
in the generalized FEM. A detailed formulation of the proposed GFEM ${ }^{\mathrm{gl}}$ for 3-D crack growth is presented in Section 3. Numerical examples demonstrating the approximating properties of the proposed enrichment functions and the computational performance of the methodology are presented in Section 4. The main conclusions are presented in Section 5.

\section{Generalized Finite Element Method: A Summary}

The generalized FEM [2, 3, 17, 40, 54] is an instance of the so-called partition of unity method (PUM), which has its origins in the works of Babuška et al. $[4,3,34]$ and Duarte and Oden [14, 15, 16, 12, 40]. The generalized FEM (GFEM) denotes a PUM with the partition of unity provided by Lagrangian finite element shape functions. The same method is also known as the extended FEM (XFEM) [5, 37]. Recent reviews of generalized/extended FEMs along with a brief history on their development can be found in $[6,23]$.

Generalized FEM approximation spaces (i.e., trial spaces) consist of three components: (a) patches or clouds, (b) a partition of unity, and (c) the patch or cloud approximation spaces. We describe these components as follows:

(a) Patch or Cloud $\omega_{\alpha}$ : In the generalized finite element method, a cloud $\omega_{\alpha}$ is given by the union of the finite elements sharing node $\alpha$ of the finite element mesh covering the domain of interest $\Omega$. The set $\left\{\omega_{\alpha}\right\}_{\alpha=1}^{N}$, in a finite element mesh with $N$ nodes, is an open cover of $\Omega$, i.e., $\Omega=$ $\cup_{\alpha=1}^{N} \omega_{\alpha}$.

(b) Partition of Unity Subordinate to the Cover $\left\{\omega_{\alpha}\right\}_{\alpha=1}^{N}$ : The Lagrangian finite element shape functions $\varphi_{\alpha}, \alpha=1, \ldots, N$, of the finite element mesh covering the domain of interest $\Omega$ constitute a partition of unity, i.e., $\sum_{\alpha=1}^{N} \varphi_{\alpha}(x)=1$ for all $x$ in $\Omega$. This is a key property used in partition of unity methods.

(c) Cloud Approximation Spaces $\chi_{\alpha}$ : To each cloud $\omega_{\alpha}$, we associate a $D_{L}(\alpha)$-dimensional space $\chi_{\alpha}$ of functions defined on $\omega_{\alpha}$, namely,

$$
\chi_{\alpha}=\operatorname{span}\left\{L_{\alpha i}, 1 \leq i \leq D_{L}(\alpha), L_{\alpha i} \in H^{1}\left(\omega_{\alpha}\right)\right\} .
$$

The basis functions $L_{\alpha i}$ above are also known as enrichment functions. A cloud approximation $\boldsymbol{u}_{\alpha}^{h p}(\boldsymbol{x}) \in \chi_{\alpha}$ of $\left.\boldsymbol{u}\right|_{\omega_{\alpha}}$ - the restriction to $\omega_{\alpha}$ of a function $\boldsymbol{u}$ defined on $\Omega$ - can be written as

$$
\boldsymbol{u}_{\alpha}^{h p}(\boldsymbol{x})=\sum_{i=1}^{D_{L}} \underline{\boldsymbol{u}}_{\alpha i} L_{\alpha i}(\boldsymbol{x})
$$

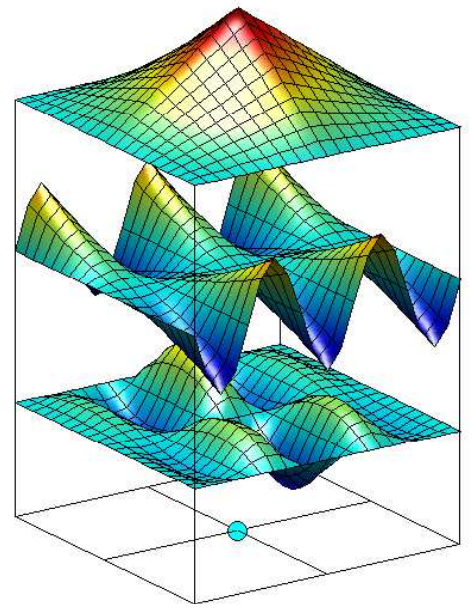

Fig. 1 Construction of a generalized FEM shape function. Here, $\varphi_{\alpha}$ is the function at the top, $L_{\alpha i}$ is the function in the middle, and the generalized FE shape function, $\phi_{\alpha i}$, is shown at the bottom

where $\underline{\boldsymbol{u}}_{\alpha i}, i=1, \ldots, D_{L}(\alpha)$, are degrees of freedom.

The trial space for the GFEM is given by

$$
\boldsymbol{X}(\Omega) \equiv \sum_{\alpha=1}^{N} \varphi_{\alpha} \chi_{\alpha}=\operatorname{span}\left\{\phi_{\alpha i}=\varphi_{\alpha} L_{\alpha i}, 1 \leq i \leq D_{L}(\alpha), 1 \leq \alpha \leq N\right\}
$$

The function

$$
\phi_{\alpha i}(\boldsymbol{x})=\varphi_{\alpha}(\boldsymbol{x}) L_{\alpha i}(\boldsymbol{x}) \quad(\text { no summation on } \alpha),
$$

where $\alpha$ is a node in the finite element mesh, is called a GFEM shape function. Figure 1 illustrates the construction of GFEM shape functions in a two-dimensional domain. 
A GFEM approximation $\boldsymbol{u}^{h p}(\boldsymbol{x}) \in \boldsymbol{X}(\Omega)$ of a vector value function $\boldsymbol{u}$ can be written as

$$
\begin{aligned}
\boldsymbol{u}^{h p}(\boldsymbol{x}) & =\sum_{\alpha=1}^{N} \sum_{i=1}^{D_{L}} \underline{\boldsymbol{u}}_{\alpha i} \phi_{\alpha i}(\boldsymbol{x})=\sum_{\alpha=1}^{N} \sum_{i=1}^{D_{L}} \underline{\boldsymbol{u}}_{\alpha i} \varphi_{\alpha}(\boldsymbol{x}) L_{\alpha i}(\boldsymbol{x}) \\
& =\sum_{\alpha=1}^{N} \boldsymbol{\varphi}_{\alpha}(\boldsymbol{x}) \sum_{i=1}^{D_{L}} \underline{\boldsymbol{u}}_{\alpha i} L_{\alpha i}(\boldsymbol{x})=\sum_{\alpha=1}^{N} \boldsymbol{\varphi}_{\alpha}(\boldsymbol{x}) \boldsymbol{u}_{\alpha}^{h p}(\boldsymbol{x}) .
\end{aligned}
$$

The enrichment functions in $\chi_{\alpha}$ must be chosen carefully to mimic the properties of the functions to be approximated in $\omega_{\alpha}$. In [46, 47], we present high order enrichment functions for 3-D fracture problems that enable modeling of surface discontinuities arbitrarily located within a finite element mesh (across elements). Nonetheless, a sufficiently fine mesh must be used around the crack front to achieve acceptable accuracy [46, 47, 48]. Even though the refinement does not have to be as strong as in the standard FEM, it leads to high computational costs when simulating, for example, 3-D propagating fractures. In this paper, we present enrichment functions for propagating fractures that are numerical solutions of boundary value problems created on-the-fly during a crack growth simulation. These functions, as demonstrated in Section 4, enable the solution of 3-D fracture problems on coarse meshes and do not require the solution of the problem from scratch at each crack evolution step.

\section{Generalized FEM with Global-Local Enrichments for 3-D Propagating Fractures}

In this section, a GFEM with global-local enrichment functions $\left(\mathrm{GFEM}^{\mathrm{gl}}\right)$ for 3-D mixed-mode propagating fractures is presented. The methodology can be formulated for several classes of crack growth problems. For simplicity and without loss of generality, we focus on the case of high-cycle quasi-static fatigue crack growth in linear elastic materials. The problem consists of a 3-D body subjected to cyclic loading and with an initial crack surface $S_{1}$ as illustrated in Figure 2(a). We assume that the stress state around the crack front can be fully characterized by linear elastic fracture mechanics and that the cyclic load has constant amplitude (cf. Figure 2(b)). The notation used in the GFEM presented here is illustrated in Figure 3. The next sections describe the methodology in detail.

\subsection{Formulation of Coarse-Scale Problem}

Consider the domain $\bar{\Omega}=\Omega \cup \partial \Omega \subset \boldsymbol{R}^{3}$. The boundary is decomposed as $\partial \Omega=\partial \Omega^{u} \cup \partial \Omega^{\sigma}$ with $\partial \Omega^{u} \cap$ $\partial \Omega^{\sigma}=\emptyset$. Figure 2(a) illustrates these definitions.

The strong form of the equilibrium and constitutive equations are given by

$$
\nabla \cdot \boldsymbol{\sigma}=\mathbf{0} \quad \boldsymbol{\sigma}=\boldsymbol{C}: \boldsymbol{\varepsilon} \quad \text { in } \Omega
$$

where $\boldsymbol{C}$ is Hooke's tensor. The following boundary conditions are prescribed on $\partial \Omega$

$$
\boldsymbol{u}=\overline{\boldsymbol{u}} \text { on } \partial \Omega^{u} \quad \boldsymbol{\sigma} \cdot \boldsymbol{n}=\overline{\boldsymbol{t}} \text { on } \partial \Omega^{\sigma}
$$

where $\boldsymbol{n}$ is the outward unit normal vector to $\partial \Omega^{\sigma}$ and $\overline{\boldsymbol{t}}$ and $\overline{\boldsymbol{u}}$ are prescribed tractions and displacements, respectively.

Let $\boldsymbol{u}_{G}^{0}$ denote the generalized or standard FEM solution of the problem defined by (5) and (6). This is hereafter denoted as the initial or uncracked global problem. The crack surface shown in Figure 2(a) is not considered when solving this problem. The approximation $\boldsymbol{u}_{G}^{0}$ is the solution of the following problem: 


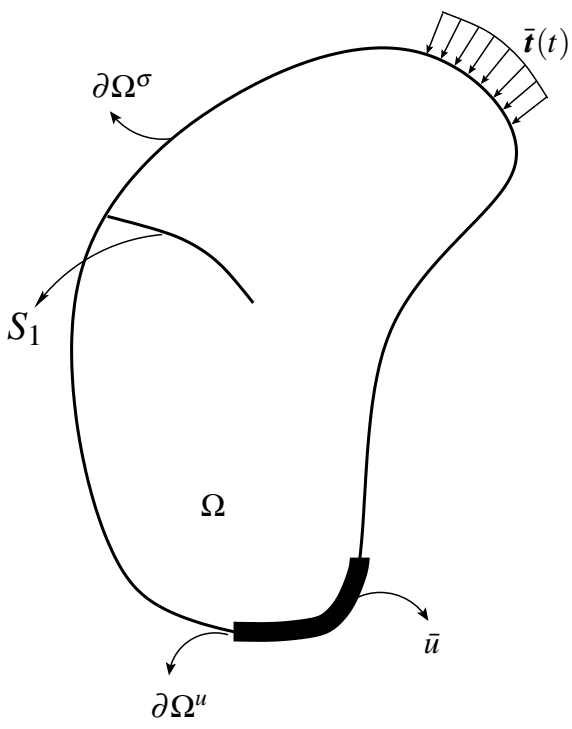

(a)

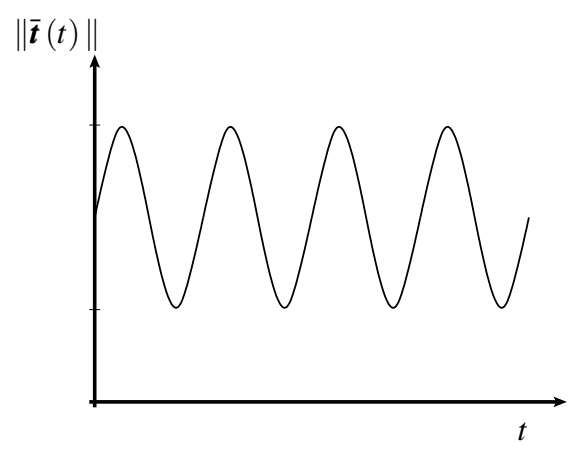

(b)

Fig. 2 Model problem and cyclic loading applied to the body. The crack surface is not considered when solving the initial global problem (7)

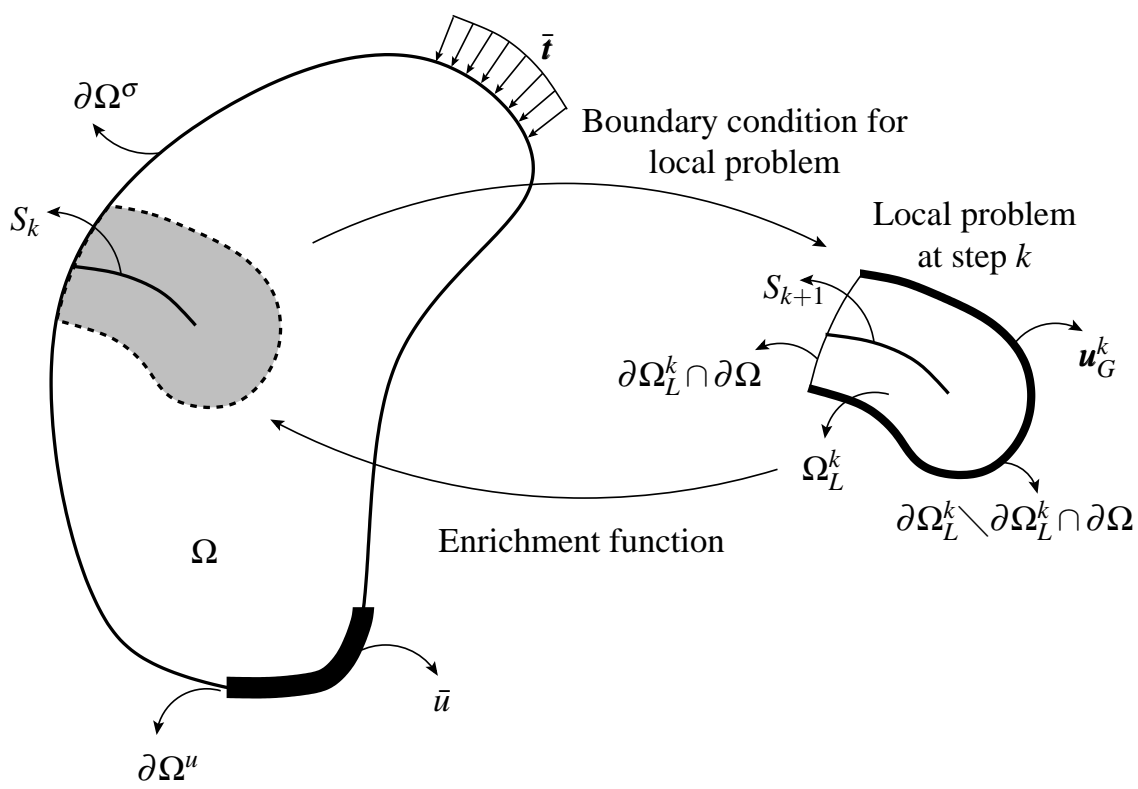

Fig. 3 Notation used in the description of the generalized FEM with global-local enrichments (GFEM ${ }^{\mathrm{gl}}$ ) for 3-D propagating fractures. The global solution $\boldsymbol{u}_{G}^{k}$ at a crack propagation step $k$ provides boundary conditions for a finescale problem defined in a neighborhood $\Omega_{L}^{k}$ of the crack surface $S_{k+1}$. The solution of this problem, in turn, is used as enrichment function for the global solution space at crack propagation step $k+1$

Find $\boldsymbol{u}_{G}^{0} \in \boldsymbol{X}_{G}^{0}(\Omega) \subset H^{1}(\Omega)$, such that $\forall \boldsymbol{v}_{G}^{0} \in \boldsymbol{X}_{G}^{0}(\Omega)$

$$
\begin{aligned}
\int_{\Omega} \boldsymbol{\sigma}\left(\boldsymbol{u}_{G}^{0}\right): \boldsymbol{\varepsilon}\left(\boldsymbol{v}_{G}^{0}\right) d \boldsymbol{x} & +\eta \int_{\partial \Omega^{u}} \boldsymbol{u}_{G}^{0} \cdot \boldsymbol{v}_{G}^{0} d \boldsymbol{s} \\
& =\int_{\partial \Omega^{\sigma}} \overline{\boldsymbol{t}} \cdot \boldsymbol{v}_{G}^{0} d \boldsymbol{s}+\eta \int_{\partial \Omega^{u}} \overline{\boldsymbol{u}} \cdot \boldsymbol{v}_{G}^{0} d \boldsymbol{s}
\end{aligned}
$$


where $\boldsymbol{X}_{G}^{0}(\Omega)$ is a discretization of $H^{1}(\Omega)$, a Hilbert space defined on $\Omega$, built with generalized or standard FEM shape functions. In this paper, the GFEM presented in [17] is used and the space $\boldsymbol{X}_{G}^{0}(\Omega)$ is given by (see also (3))

$$
\boldsymbol{X}_{G}^{0}(\Omega)=\left\{\boldsymbol{u}^{h p}=\sum_{\alpha=1}^{N} \varphi_{\alpha}(\boldsymbol{x}) \hat{\boldsymbol{u}}_{\alpha}^{h p}(\boldsymbol{x}): \hat{\boldsymbol{u}}_{\alpha}^{h p}(\boldsymbol{x})=\sum_{i=1}^{\hat{D}_{L}} \underline{\hat{\boldsymbol{u}}}_{\alpha i} \hat{L}_{\alpha i}(\boldsymbol{x})\right\}
$$

where $\underline{\hat{u}}_{\alpha i}, \alpha=1, \ldots, N, i=1, \ldots, \hat{D}_{L}$ are nodal degrees of freedom and $\hat{D}_{L}$ is the dimension of a set of polynomial enrichment functions, $\hat{L}_{\alpha i}(\boldsymbol{x})$, of a degree less than or equal to $p-1$. Details can be found, for example, in [17] or Section 3.2 of [46]. Space $\boldsymbol{X}_{G}^{0}(\Omega)$ can also be defined using standard polynomial FEM shape functions since cracks are not discretized in the initial global problem.

The parameter $\eta$ in (7) is a penalty parameter based on Young's modulus and the Jacobian of elements with a face on $\partial \Omega^{u}$.

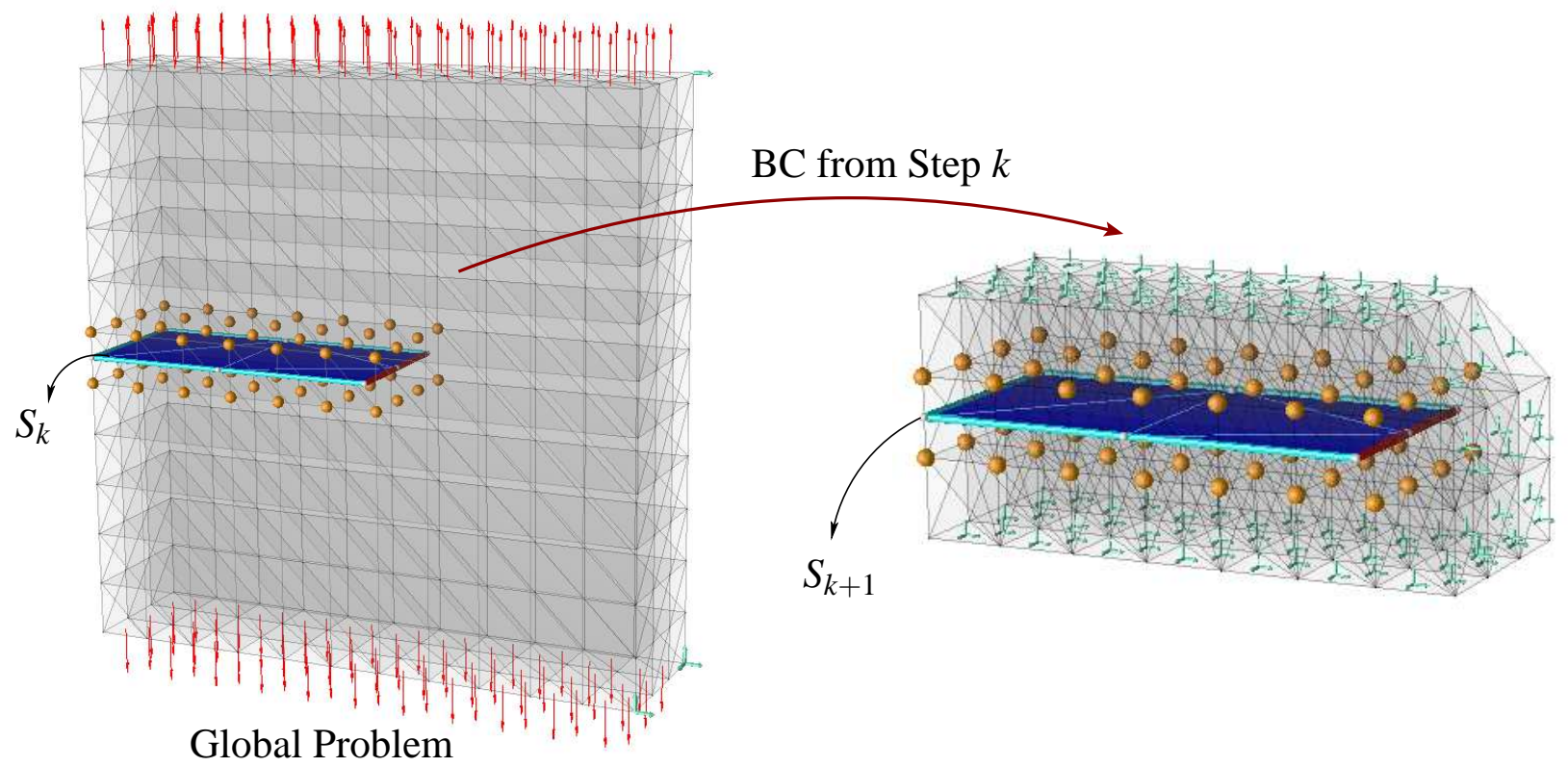

Fig. 4 Illustration of the GFEM $^{\mathrm{gl}}$ for crack propagation. The figure shows an edge-cracked plate under mode $I$ loading. The solution computed on the coarse mesh provides boundary conditions for the extracted local domain $\Omega_{L}^{k}$ in a neighborhood of the crack (on element faces with green arrows). The spheres indicate seed nodes used in the definition of the local problem domain. Further details on the definition of $\Omega_{L}^{k}$ are provided in Appendix B

\subsection{Enriched Coarse-Scale Problem}

Global-local enrichment functions for propagating fractures are able to represent fine-scale responses on coarse macro-scale finite element meshes and to fully account for interactions among scales. The formulation of the coarse-scale problems enriched with these functions is provided in this section, while their definition and computation are presented in Section 3.3. The coarse-scale solution spaces used in this section are defined in Section 3.4.

Let $\boldsymbol{u}_{G}^{k}$ denote a generalized FEM approximation of the global problem illustrated in Figure 3. For simplicity of notation, we assume that a single stress-free crack surface $S_{k}$ with front $\Gamma_{k}$ exists in $\Omega$ at crack evolution step $k, k \geq 1$. The initial crack surface configuration corresponds to $S_{1}$, and $k=0$ refers to the initial (uncracked) global problem (see also Figure 7). The approximation $\boldsymbol{u}_{G}^{k}$ is the solution of the following problem: 
Find $\boldsymbol{u}_{G}^{k} \in \boldsymbol{X}_{G}^{k}(\Omega) \subset H^{1}(\Omega)$, such that $\forall \boldsymbol{v}_{G}^{k} \in \boldsymbol{X}_{G}^{k}(\Omega)$

$$
\begin{aligned}
\int_{\Omega} \boldsymbol{\sigma}\left(\boldsymbol{u}_{G}^{k}\right): \boldsymbol{\varepsilon}\left(\boldsymbol{v}_{G}^{k}\right) d \boldsymbol{x} & +\eta \int_{\partial \Omega^{u}} \boldsymbol{u}_{G}^{k} \cdot \boldsymbol{v}_{G}^{k} d \boldsymbol{s} \\
& =\int_{\partial \Omega^{\sigma}} \overline{\boldsymbol{t}} \cdot \boldsymbol{v}_{G}^{k} d \boldsymbol{s}+\eta \int_{\partial \Omega^{u}} \overline{\boldsymbol{u}} \cdot \boldsymbol{v}_{G}^{k} d \boldsymbol{s}
\end{aligned}
$$

where $\boldsymbol{X}_{G}^{k}(\Omega) \subset H^{1}(\Omega)$ is the generalized FEM space at crack propagation step $k, k \geq 1$ (cf. Section 3.4). The enrichment functions in $\boldsymbol{X}_{G}^{k}(\Omega)$ are defined in cloud spaces $\mathscr{I}_{\alpha}$ and have to be computed; we describe a fine-scale problem in the next subsection to achieve this goal. The mesh used to solve Problem (9) is typically a coarse quasi-uniform mesh, regardless of the presence of cracks in the domain. Figures 4 and 5 illustrate one such discretization. Problem (9) leads to a system of linear equations for the unknown degrees of freedom of $\boldsymbol{u}_{G}^{k}$. The only difference between problem statements (7) and (9) is the GFEM spaces. In the first case, cracks are not discretized, while in second case, they are discretized using global-local enrichment functions built on-the-fly as described below.

\subsection{Fine-Scale Problems}

The GFEM ${ }^{\mathrm{gl}}$ for propagating fractures involves the solution of a local boundary value problem defined in a neighborhood $\Omega_{L}^{k}$ of the crack surface $S_{k+1}$ and subjected to boundary conditions provided by the coarse-scale GFEM solution $\boldsymbol{u}_{G}^{k}, k \geq 0$. Here, $S_{1}$ represents the initial crack configuration before starting its propagation. This is illustrated in Figures 3 and 4. Details on the definition of $\Omega_{L}^{k}$ are provided in Appendix B. Let $\boldsymbol{u}_{G}^{k}$ denote the global approximation computed using (7) for $k=0$ or (9) for $k \geq 1$. The following fine-scale problem on $\Omega_{L}^{k} \subset \Omega$ is solved to find global-local enrichment functions for the GFEM space $\boldsymbol{X}_{G}^{k+1}(\Omega)$ :

Find $\boldsymbol{u}_{L}^{k} \in \boldsymbol{X}_{L}^{k}\left(\Omega_{L}^{k}\right) \subset H^{1}\left(\Omega_{L}^{k}\right)$, such that $\forall \boldsymbol{v}_{L}^{k} \in \boldsymbol{X}_{L}^{k}\left(\Omega_{L}^{k}\right)$

$$
\begin{aligned}
\int_{\Omega_{L}^{k}} \boldsymbol{\sigma}\left(\boldsymbol{u}_{L}^{k}\right): \boldsymbol{\varepsilon}\left(\boldsymbol{v}_{L}^{k}\right) d \boldsymbol{x} & +\eta \int_{\partial \Omega_{L}^{k} \backslash\left(\partial \Omega_{L}^{k} \cap \partial \Omega^{\sigma}\right)} \boldsymbol{u}_{L}^{k} \cdot \boldsymbol{v}_{L}^{k} d s \\
& =\int_{\partial \Omega_{L}^{k} \cap \partial \Omega^{\sigma}} \overline{\boldsymbol{t}} \cdot \boldsymbol{v}_{L}^{k} d s+\eta \int_{\partial \Omega_{L}^{k} \cap \partial \Omega^{u}} \overline{\boldsymbol{u}} \cdot \boldsymbol{v}_{L}^{k} d s \\
& +\eta \int_{\partial \Omega_{L}^{k} \backslash\left(\partial \Omega_{L}^{k} \cap \partial \Omega\right)} \boldsymbol{u}_{G}^{k} \cdot \boldsymbol{v}_{L}^{k} d s
\end{aligned}
$$

where $\boldsymbol{X}_{L}^{k}\left(\Omega_{L}^{k}\right)$ is a discretization of $H^{1}\left(\Omega_{L}^{k}\right)$ using the GFEM shape functions presented in [46, 47]. The mesh used in $\Omega_{L}^{k}$ does not fit the crack surface. Furthermore, since this mesh is usually much finer than the one used in the global problem, they do not match at $\partial \Omega_{L}^{k}$. The local mesh is created by bisecting elements copied from the global mesh [13]. The integrals over $\Omega_{L}^{k}$ and $\partial \Omega_{L}^{k}$ are performed using the fine scale mesh as described in Section 3.5 of [30]. The computation of the global solution $\boldsymbol{u}_{G}^{k}$ at an integration point on $\partial \Omega_{L}^{k} \backslash\left(\partial \Omega_{L}^{k} \cap \partial \Omega\right)$ basically requires the computation of the corresponding master coordinates at the global element face on $\partial \Omega_{L}^{k} \backslash\left(\partial \Omega_{L}^{k} \cap \partial \Omega\right)$ and the evaluation of $\boldsymbol{u}_{G}^{k}$ at these coordinates. Figure 5 shows an $h p$-GFEM discretization on a local domain $\Omega_{L}^{k}$ containing crack surface $S^{k+1}$.

A key aspect of Problem (10) is the use of the coarse-scale solution at simulation step $k, \boldsymbol{u}_{G}^{k}$, as boundary condition on $\partial \Omega_{L}^{k} \backslash\left(\partial \Omega_{L}^{k} \cap \partial \Omega\right)$. Exact boundary conditions are prescribed elsewhere on $\partial \Omega_{L}^{k}$. Other types of boundary conditions such as spring or traction boundary conditions can also be applied on $\partial \Omega_{L}^{k} \backslash\left(\partial \Omega_{L}^{k} \cap\right.$ $\partial \Omega)[30]$. 


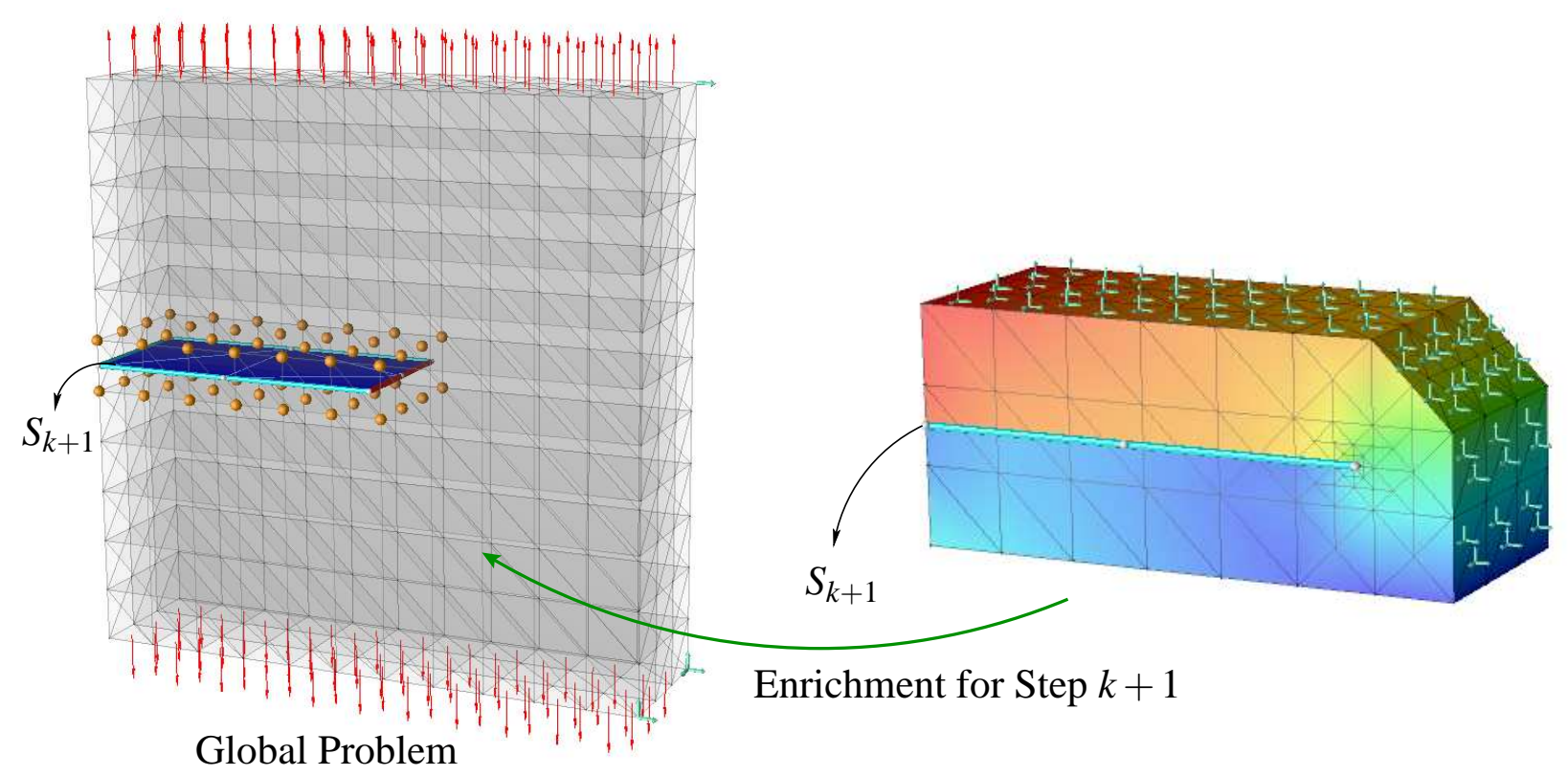

Fig. 5 Hierarchical enrichment of the coarse global mesh with local solutions computed with the $h p$-GFEM on the local domain. Only three degrees of freedom are added to these global nodes (shown with brown spheres in the figure). These enrichments are used to approximate the global solution in the neighborhood of the crack

\subsection{Enriched Global Spaces}

The solution, $\boldsymbol{u}_{L}^{k}$, of the fine-scale problem defined in (10) is used to build generalized FEM shape functions defined on the coarse-scale (global) mesh:

$$
\boldsymbol{\phi}_{\alpha}^{k+1}(\boldsymbol{x}):=\varphi_{\alpha}(\boldsymbol{x}) \boldsymbol{u}_{L}^{k}(\boldsymbol{x})
$$

where the partition of unity function, $\varphi_{\alpha}$, is provided by a global, coarse, FE mesh and $\boldsymbol{u}_{L}^{k}$ has the role of an enrichment or basis function for the cloud space $\chi_{\alpha}\left(\omega_{\alpha}\right)$. Hereafter, $\boldsymbol{u}_{L}^{k}$ is denoted as a global-local enrichment function and the function defined above is denoted as a global-local GFEM shape function. The global GFEM space containing shape functions $\boldsymbol{\phi}_{\alpha}^{k+1}$ is denoted as $\boldsymbol{X}_{G}^{k+1}(\Omega)$ and is given by

$$
\begin{aligned}
\boldsymbol{X}_{G}^{k+1}(\Omega) & =\{\boldsymbol{u}^{h p}=\underbrace{\sum_{\alpha=1}^{N} \varphi_{\alpha}(\boldsymbol{x}) \hat{\boldsymbol{u}}_{\alpha}^{h p}(\boldsymbol{x})}_{\text {coarse-scale approx. }}+\underbrace{\sum_{\beta \in \mathscr{I}_{g l}^{k+1}} \varphi_{\beta}(\boldsymbol{x}) \boldsymbol{u}_{\beta}^{g l(k)}(\boldsymbol{x})}_{\text {fine-scale approx. }}\} \\
& =\boldsymbol{X}_{G}^{0}(\Omega) \bigcup\left\{\varphi_{\beta}(\boldsymbol{x}) \boldsymbol{u}_{\beta}^{g l(k)}(\boldsymbol{x}), \beta \in \mathscr{I}_{g l}^{k+1}\right\}
\end{aligned}
$$

where $\mathscr{I}_{g l}^{k+1}$ is the index set of nodes (i.e., clouds) at crack growth step $k+1$ enriched with global-local enrichment functions $\boldsymbol{u}_{L}^{k}, \hat{\boldsymbol{u}}_{\alpha}^{h p}$ is defined in (8) and

$$
\boldsymbol{u}_{\beta}^{g l(k)}=\left[\begin{array}{c}
\underline{u}_{\beta 1} u_{L 1}^{k}(\boldsymbol{x}) \\
\underline{u}_{\beta 2} u_{L 2}^{k}(\boldsymbol{x}) \\
\underline{u}_{\beta 3} u_{L 3}^{k}(\boldsymbol{x})
\end{array}\right]
$$




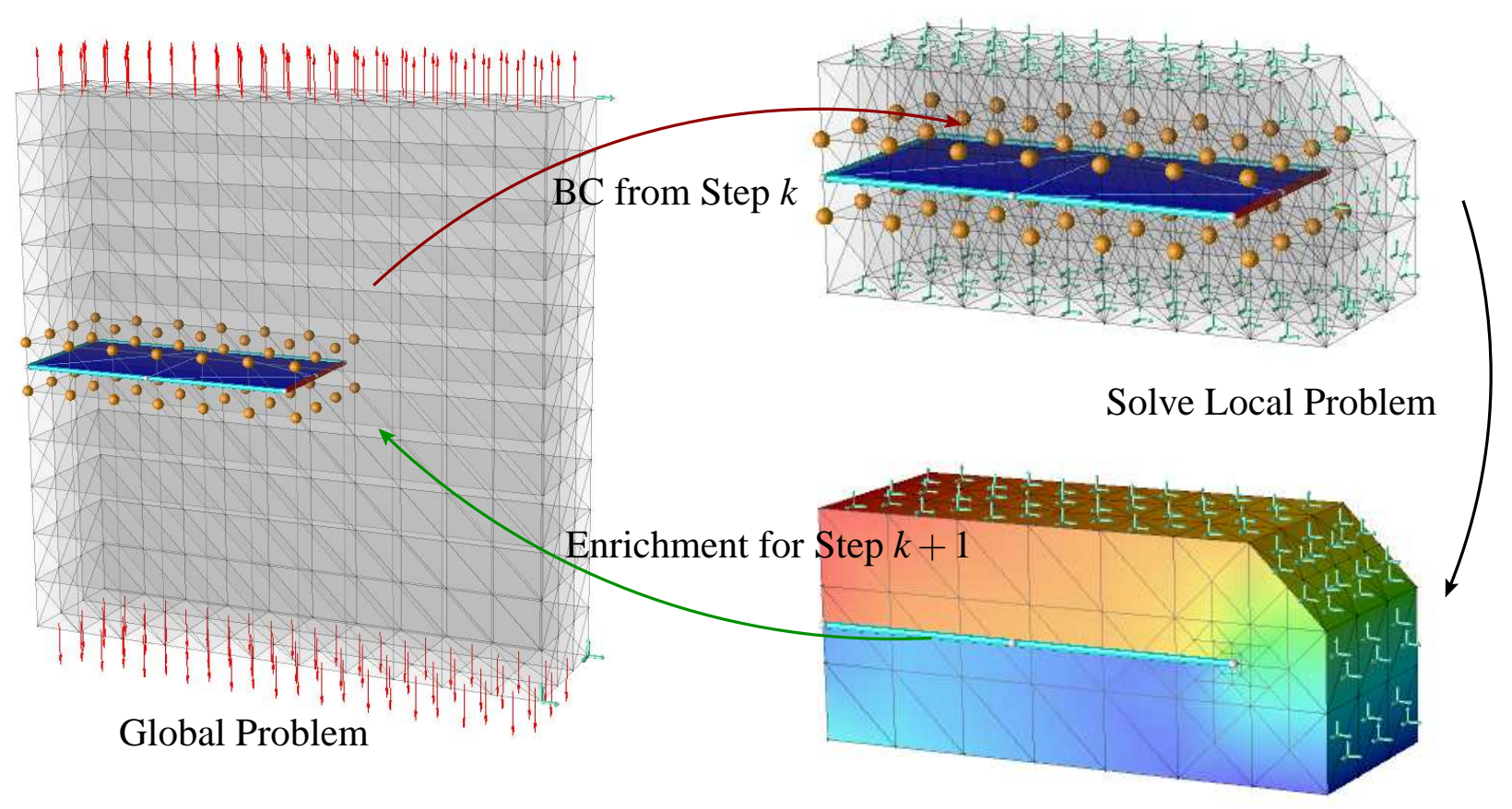

Fig. 6 Illustration of the GFEM $^{\text {gl }}$ for crack propagation. In the figure, the local domain is selected on-the-fly such that it contains the entire crack surface

where $\underline{u}_{\beta j}, \beta \in \mathscr{I}_{g l}^{k+1}, j=1,2,3$, are degrees of freedom and $u_{L j}^{k}(\boldsymbol{x}), j=1,2,3$, are global Cartesian components of the displacement vector $\boldsymbol{u}_{L}^{k}$. Space $\boldsymbol{X}_{G}^{k+1}(\Omega)$ is the space $\boldsymbol{X}_{G}^{0}(\Omega)$ augmented with global-local enrichment functions computed at crack simulation step $k$. Figure 5 illustrates the enrichment of a global mesh with the solution of a local problem. Global nodes in the index set $\mathscr{I}_{g l}^{k+1}$ are shown in the figure with brown spheres.

The coarse-scale problem defined in (9) is solved for $\boldsymbol{u}_{G}^{k+1} \in \boldsymbol{X}_{G}^{k+1}(\Omega)$ and the procedure is repeated at each crack evolution step. The GFEM $^{\mathrm{gl}}$ for crack propagation is illustrated in Figures 3 and 6 . The global solution provides boundary conditions for fine-scale problems, while their solutions are used as enrichment functions for the coarse-scale problem through the partition of unity framework of the GFEM.

The numerical integration of the GFEM shape functions defined in (11) cannot be performed by the coarse scale mesh. They can, however, be integrated efficiently and accurately using the elements from the local mesh used for the computation of $\boldsymbol{u}_{L}^{k}$ since they are nested in the coarse scale elements [13, 30]. This procedure is analogous to the concept of integration elements broadly used for the numerical integration in the extended FEM [5, 37]. The elements used in the numerical integration of the weak form are also useful for the visualization of results of the enriched global problem. One example is shown in Figure 14.

\subsection{Crack Growth Algorithm with the GFEMgl}

This section presents an algorithm for the simulation of fatigue crack growth using the GFEM ${ }^{\mathrm{gl}}$ presented above. The algorithm consists of an incremental process in which at each step, a small crack advance is prescribed based on the solution of a linear elastic fracture mechanics problem.

Let the crack surface at crack evolution step $k$ be denoted by $S_{k}$. The initial crack surface configuration corresponds to $S_{1}$. The GFEM ${ }^{\mathrm{gl}}$ algorithm for crack growth consists of the following steps:

1. Solve the initial coarse-scale problem without cracks: Compute $\boldsymbol{u}_{G}^{0}$ using (7). Keep the factorization of 
the global stiffness matrix $\left(\boldsymbol{K}^{0}\right)^{-1}$ so it can be re-used in the solution of the enriched global problems as described in Appendix C.

2. For each crack simulation step $k, k=0, \ldots, N_{\text {steps }}$, do:

(a) Using $S_{k+1}$, select the global elements that define the local problem domain, $\Omega_{L}^{k}$, at this step. Details on this procedure are presented in Appendix B.

(b) Compute the local problem solution, $\boldsymbol{u}_{L}^{k}$, using (10) with crack surface $S_{k+1}$ and with boundary conditions on $\partial \Omega_{L}^{k} \backslash\left(\partial \Omega_{L}^{k} \cap \partial \Omega\right)$ provided by the global solution $\boldsymbol{u}_{G}^{k}$.

(c) Compute the solution of the enriched global problem $\boldsymbol{u}_{G}^{k+1} \in \boldsymbol{X}_{G}^{k+1}(\Omega)$ using (9) with crack surface $S_{k+1}$. The global solution space $\boldsymbol{X}_{G}^{k+1}(\Omega)$ is defined in (12) and uses the local solution $\boldsymbol{u}_{L}^{k}$ as enrichment function (cf. Section 3.4). The algorithm presented in Appendix C is used in the solution of the linear system of equations associated with Problem (9).

(d) If $k>0$, compute crack surface configuration $S_{k+2}$ using global solution $\boldsymbol{u}_{G}^{k+1}$ (cf. Appendix A for details). The crack surface is not updated at crack step $k=0$ since the boundary conditions for the local problem defined on $\Omega_{L}^{0}$ are provided by the initial (uncracked) global solution $\boldsymbol{u}_{G}^{0}$.

(e) If $k=N_{\text {steps }}$, stop; otherwise, set $k=k+1$ and go to step (2a).

Figure 6 illustrates the interactions between coarse and fine scales on an edge-crack panel and Figure 7 illustrates the first two steps of the above algorithm. A key feature of the methodology is the use of the global solution at simulation step $k, \boldsymbol{u}_{G}^{k}$, to build the solution space for the next simulation step, i.e., the $\mathrm{GFEM}^{\mathrm{gl}}$ space $\boldsymbol{X}_{G}^{k+1}(\Omega)$ containing the GFEM ${ }^{\mathrm{gl}}$ solution $\boldsymbol{u}_{G}^{k+1}$. The coarse-scale solution $\boldsymbol{u}_{G}^{k}$, is used as the boundary condition on $\partial \Omega_{L}^{k} \backslash\left(\partial \Omega_{L}^{k} \cap \partial \Omega\right)$ for the fine-scale problem (10) instead of the unknown exact solution at step $k+1$. As a result, the error of $\boldsymbol{u}_{L}^{k}$ depends not only on the discretization used in the local domain $\Omega_{L}$, but also on how much the solution of the problem changes at $\partial \Omega_{L}^{k} \backslash\left(\partial \Omega_{L}^{k} \cap \partial \Omega_{G}\right)$ between crack steps. Since crack increments must be small for accurate results, it is reasonable to assume that the change in the solution between crack steps is also small. The examples presented in Section 4 show that the effect of inexact boundary conditions on $\partial \Omega_{L}^{k} \backslash\left(\partial \Omega_{L}^{k} \cap \partial \Omega_{G}\right)$ on the accuracy of the enriched global problem is small. Detailed error analysis of the GFEM $^{\mathrm{gl}}$ for crack growth will be presented elsewhere. Furthermore, this effect can be controlled using the iterative improvement of boundary conditions proposed in [41, 42]. The effect of the inexact boundary conditions on the accuracy of $\boldsymbol{u}_{L}^{k}$ can be addressed by repeating the procedure illustrated in Figure 6 at each crack simulation step:

1. Use the solution of the global problem $\boldsymbol{u}_{G}^{k+1} \in \boldsymbol{X}_{G}^{k+1}(\Omega)$ as boundary conditions for the fine-scale problem (10) defined on $\Omega_{L}^{k}$.

2. Update global shape functions (11) and global solution space $\boldsymbol{X}_{G}^{k+1}(\Omega)$.

3. Solve the enriched coarse-scale problem (9) for $\boldsymbol{u}_{G}^{k+1} \in \boldsymbol{X}_{G}^{k+1}(\Omega)$.

4. Go to step 1 if the accuracy of $\boldsymbol{u}_{G}^{k+1}$ is not acceptable; proceed to the next crack step otherwise.

In this paper, this iterative improvement is performed only once at the first crack simulation step and only used at step (2d) of the algorithm described above. This strategy is illustrated using a dashed arrow in Figure 7. Hereafter, this strategy is denoted as Improvement at Starting Step (ISS). Its effect on the accuracy of global quantities like strain energy is analyzed in Section 4.1. Another multi-scale method for 3-D crack growth based on a multi-grid iterative solver is presented in [50].

Another key feature of the methodology presented above is that the coarse-scale mesh is kept unchanged throughout the simulation. The enriched coarse-scale problem at any crack simulation step is solved on the 


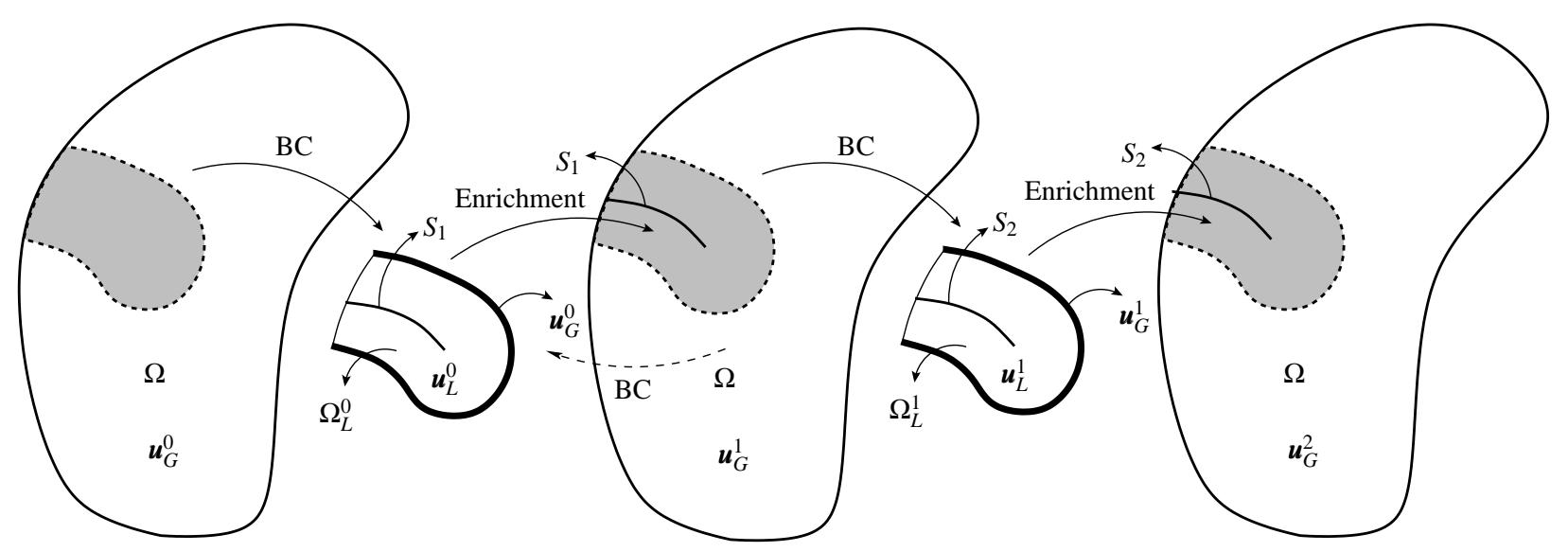

Fig. 7 Illustrations of the first two steps of the GFEM $^{\mathrm{gl}}$ for crack growth and the Improvement at Starting Step (ISS) indicated by a dashed arrow in the figure

same uncracked mesh used in the computation of $\boldsymbol{u}_{G}^{0}$. Fine-scale features are hierarchically added to the global-solution space through global-local enrichment functions. These functions allow the simulation of crack surfaces with arbitrary shape using fairly coarse global meshes. The numerical example presented in Section 4.3 also demonstrates that the GFEM $^{\mathrm{gl}}$ is more computationally efficient than the $h p$-GFEM, which is among the most efficient methods currently available in the literature.

\subsection{Alternative Strategy: Local Domain with Crack Front Only}

In the GFEM $^{\mathrm{gl}}$ described above and illustrated in Figure 6, the local domain is selected on-the-fly at each crack propagation step such that it contains the entire crack surface. Furthermore, the discontinuities and singularities of the global solution along the crack surface and crack front are approximated exclusively by the global-local enrichment functions. This is reflected in the definition of the enriched global spaces given in (12). There are several possible variations to this methodology. One of them is illustrated in Figure 8. In this case, the local domain $\Omega_{L}^{k}$ contains the entire crack front but not the entire crack surface. Clouds fully cut by the crack surface but not in the set enriched with local solution $\boldsymbol{u}_{L}^{k}$, i.e., not in the set $\mathscr{I}_{g l}^{k+1}$, are enriched instead with the analytically defined discontinuous functions [46]. Let $\mathscr{I}_{\mathscr{H}}^{k+1}$ denote the index set of clouds enriched with these functions at crack growth step $k+1$. The enriched global space at crack step $k+1$ is then given by

$$
\boldsymbol{X}_{G}^{k+1}(\Omega)=\left\{\boldsymbol{u}^{h p}=\sum_{\alpha=1}^{N} \varphi_{\alpha}(\boldsymbol{x}) \hat{\boldsymbol{u}}_{\alpha}^{h p}(\boldsymbol{x})+\sum_{\beta \in \mathscr{F}_{g l}^{k+1}} \varphi_{\beta}(\boldsymbol{x}) \boldsymbol{u}_{\beta}^{g l(k)}(\boldsymbol{x})+\sum_{\gamma \in \mathscr{I}_{\mathscr{H}}^{k+1}} \varphi_{\gamma}(\boldsymbol{x}) \mathscr{H} \tilde{\boldsymbol{u}}_{\gamma}^{h p}(\boldsymbol{x})\right\}
$$

The performances of this and the previous versions of the GFEM ${ }^{\mathrm{gl}}$ are investigated in Section 4.

\section{Numerical Examples}

This section presents three numerical examples to verify and measure the computational performance of the GFEM $^{\mathrm{gl}}$ for crack growth. The problems are also solved with the $h p$-GFEM for fatigue crack growth presented in [48]. Since the $h p$-GFEM methodology is extensively verified in that paper, it is adopted as a reference. In all examples, both methods use the same polynomial enrichment $(p=3)$ and localized crack front refinement $L_{e} / a_{o}, \simeq 10^{-2}$, where $a_{o}$ is the initial crack size and $L_{e}$ is the tetrahedral element size. In 


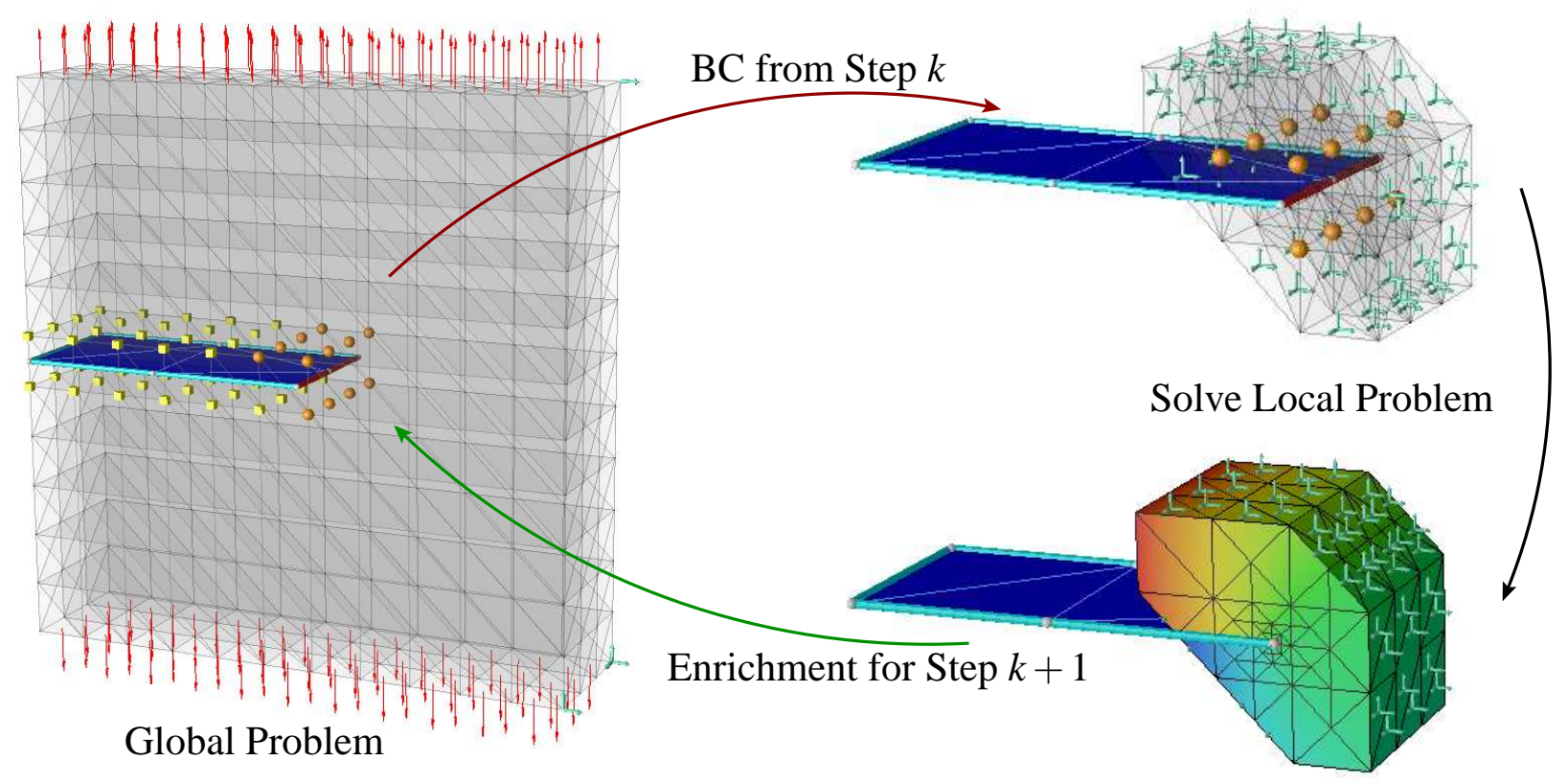

Fig. 8 Illustration of the GFEM $^{\mathrm{gl}}$ with local domains defined around the crack front only. The discontinuous global solution away from the crack front is approximated with analytically defined high-order discontinuous enrichment functions. Global nodes enriched with these functions are marked with yellow cubes and belong to the set $\mathscr{I}_{\mathscr{H}}^{k+1}$. Brown spheres in the global domain belong to the set $\mathscr{J}_{g l}^{k+1}$ and are enriched with global-local enrichment functions

the case of the GFEM ${ }^{\mathrm{gl}}$, mesh refinement is applied in local problems only. Global problems use coarse quasi-uniform meshes.

\subsection{Fatigue Crack Growth in an Edge-Cracked Plate}

As a proof of concept, this section presents a simple example of fatigue crack growth simulation using the proposed $\mathrm{GFEM}^{\mathrm{gl}}$. Consider the edge-cracked plate under cyclic uniaxial tension in the y-direction illustrated in Figure 9. The dimensions of the model are $2 h / t=b / t=4$ and $a_{o} / t=2.1$. Young's modulus and Poisson's ratio are $E=2.0 \times 10^{5} \mathrm{MPa}$ and $v=0.30$, respectively. The parameters for the cyclic load and Paris-Erdogan's equation (18) are $\sigma_{\max }=1 \mathrm{MPa}, R=0$ and $C=1.5463 \times 10^{-11} \mathrm{MPa}^{-2.1} \mathrm{~m}^{-0.05} / \mathrm{cycle}$ and $m=2.1$, respectively. This problem is solved using the algorithm described in Section 3.5. Reference values for strain energy and stress intensity factors are provided by the $h p$-GFEM for crack growth presented in [48]. The plane strain mode $I$ stress intensity factor (SIF) is also used as a reference. In the case of a finite edge-cracked plate, $K_{I}$ is given by [59]

$$
K_{I}=\sigma \sqrt{\pi a} F\left(\frac{a}{b}\right)
$$

where

$$
F\left(\frac{a}{b}\right)=\sqrt{\frac{2 b}{\pi a} \tan \left(\frac{\pi a}{2 b}\right)} \frac{0.752+2.02 \frac{a}{b}+0.37\left[1-\sin \left(\frac{\pi a}{2 b}\right)\right]^{3}}{\cos \left(\frac{\pi a}{2 b}\right)} .
$$

The plane strain SIF is compared with GFEM $^{\mathrm{gl}}$ and $h p$-GFEM values extracted at $t / 2$. A crack front increment, $\Delta a$, is prescribed at each crack step. Furthermore, the crack front is kept straight as shown in Figure 9 in order to reduce $3-\mathrm{D}$ effects. 


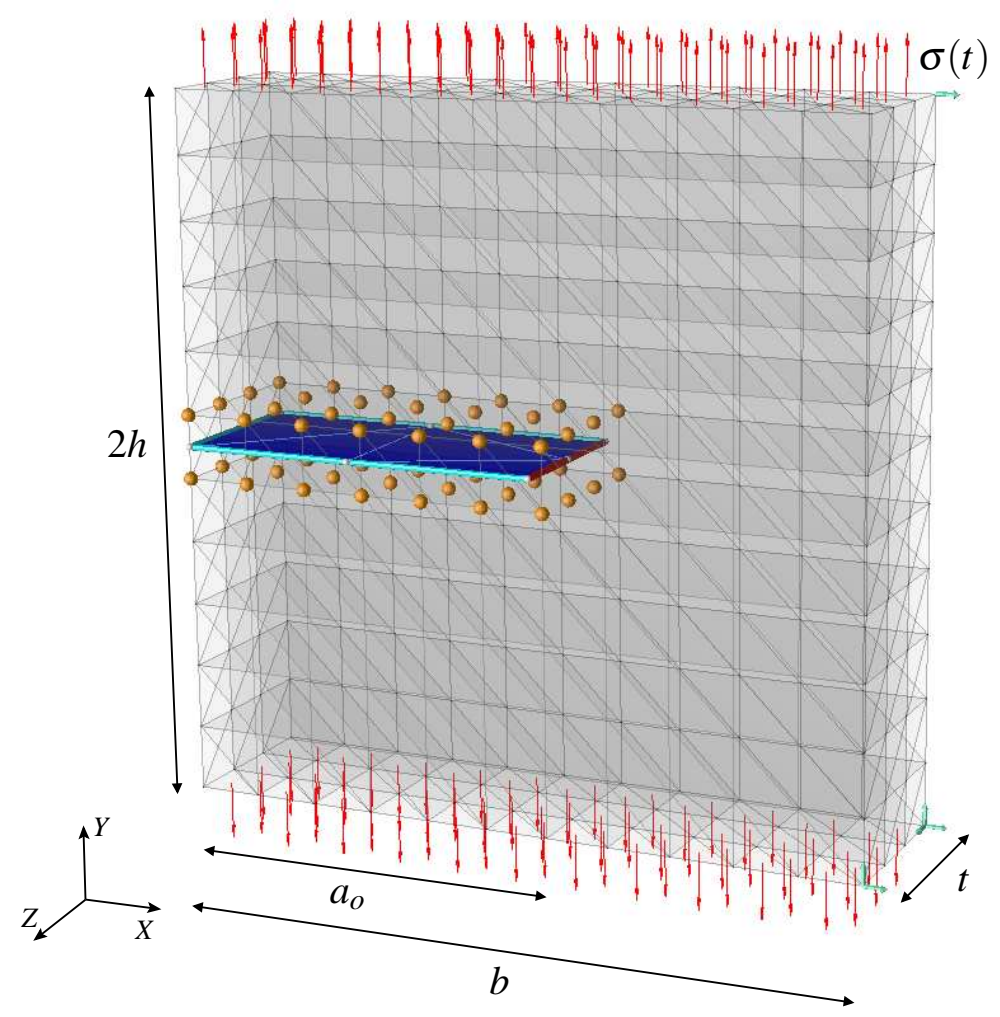

Fig. 9 Edge-cracked panel subjected to cyclic load. Brown spheres represent nodes with the global-local enrichment functions

The performance of GFEM $^{\mathrm{gl}}$ strategies illustrated in Figures 6 and 8 are compared in this example. They are hereafter denoted as Strategies 1 and 2, respectively. In the case of Strategy 1, the local problem domains are defined by global elements that intersect the crack surface and their neighbors while Strategy 2 defines local problem domains using global elements that intersect the crack front and their neighbors. Strategy 1 is used with and without the Improvement at Starting Step (ISS) described in Section 3.5. Strategy 2 does not use the Improvement at Starting Step.

Figures 10(a) and 11(a) show the evolution of the strain energy and SIF for the $h p$-GFEM and the GFEM ${ }^{\mathrm{gl}}$ with respect to crack growth length, respectively. One can observe that both methods provide virtually the same values of strain energy and SIF at all crack growth steps. Figure 10(b) plots the relative difference in strain energy of GFEM ${ }^{\mathrm{gl}}$ solutions with respect to $h p$-GFEM solutions. In the case of the $\mathrm{GFEM}^{\mathrm{gl}}$ with Strategy 2, the relative difference is always below 1.5\%, while in the case of Strategy 1 with ISS, the relative difference is below $0.5 \%$. Strategy 1 without ISS has about the same error as Strategy 1 with ISS except at the first crack propagation step. This shows that the Improvement at the Starting Step described in Section 3.5 may be important when the crack is discretized using solution space (12). Figure 11(b) shows the relative difference of SIF computed with the GFEM ${ }^{\mathrm{gl}}$ and the $h p$-GFEM with respect to the plane strain solution. All methods show a similar behavior. Strategy 1 without ISS, again, has a large relative difference at the first crack propagation step and about the same behavior as Strategy 1 with ISS at all other steps. In this example, crack growth is governed by mode $I$ only, and thus the error of Strategy 1 without the improvement of boundary conditions at the first crack step does not affect subsequent steps. However, in a mixed-mode situation, incorrect SIF values at the first crack propagation step may give an incorrect crack growth direction, leading to discrepancies in subsequent steps. The remaining examples in this paper are solved using Strategy 1 with ISS. 


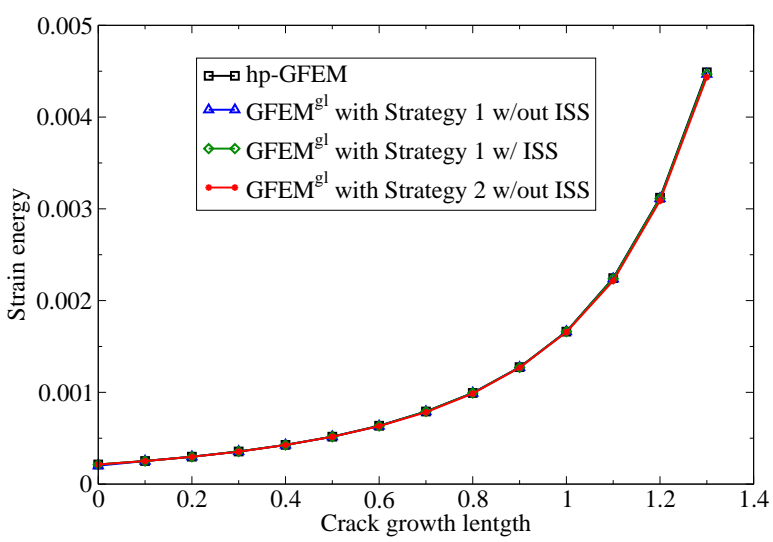

(a) Strain energy

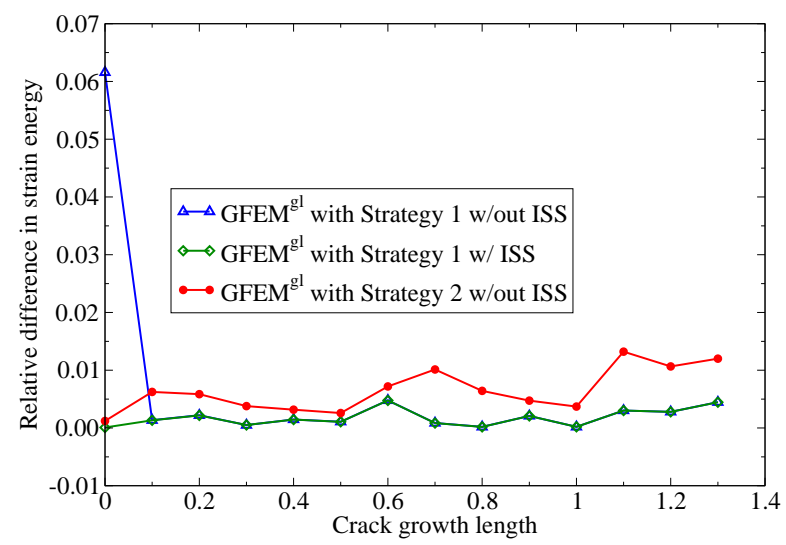

(b) Relative difference of GFEM ${ }^{\mathrm{gl}}$ solution with respect to the $h p$-GFEM solution

Fig. 10 Strain energy evolution with respect to crack growth length

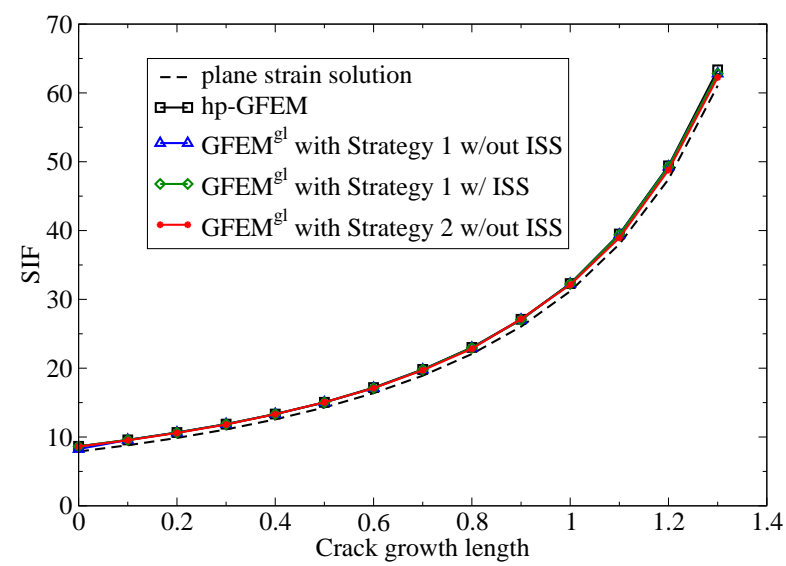

(a) SIF

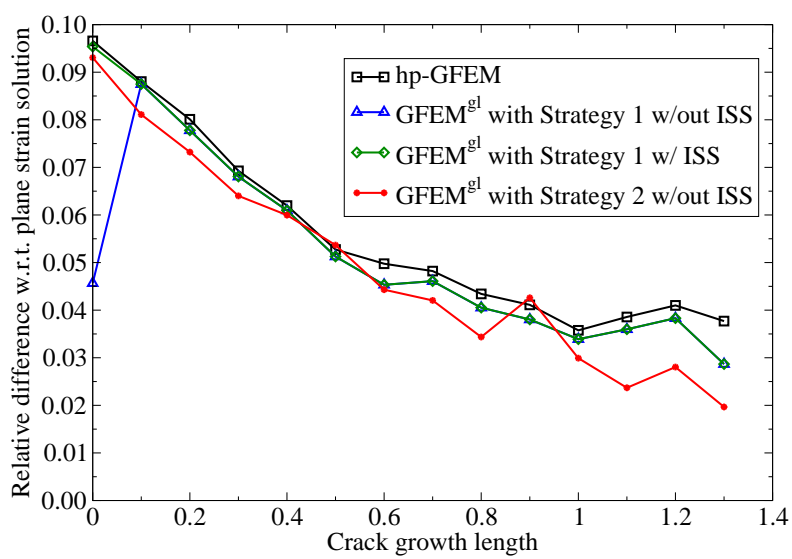

(b) Relative difference with respect to the plane strain solution

Fig. 11 SIF evolution with respect to crack growth length

In a GFEM $^{\mathrm{gl}}$ discretization, only a few global-local enrichments are added to the global problem. For instance, let us consider the first crack propagation step of the GFEM $^{\mathrm{gl}}$ simulation with Strategies 1 and 2. At this step, the global problem with Strategy 2 has a total of 18636 degrees of freedom (dofs). From this total, 17280 dofs correspond to the initial global discretization without the crack, 1320 dofs correspond to discontinuous enrichment functions, and only 36 dofs correspond to global-local enrichment functions (cf. solution space defined in (14)).

In the case of Strategy 1, the global problem at the first crack propagation step has a total of 17448 dofs: 17280 dofs correspond to continuous shape functions and only 168 dofs correspond to global-local enrichment functions (cf. solution space defined in (12)). No analytically-defined discontinuous enrichments are used in this case since the crack surface is contained in the local domain and the solution discontinuity is approximated by global-local enrichments only. This shows that Strategy 1 is clearly more efficient than Strategy 2.

Table 1 lists the range of problem sizes solved with GFEM ${ }^{\mathrm{gl}}$ and $h p$-GFEM. Both global and local problem sizes are listed in the case of $\mathrm{GFEM}^{\mathrm{gl}}$. Problem sizes are not affected by the use of the Improvement at Starting Step (ISS). One can observe that the size of global problems solved in the case of the GFEM $^{\mathrm{gl}}$ with 
either Strategy 1 or 2 are much smaller than in the case of the $h p$-GFEM. Since the accuracy of both methods is about the same, one can expect that crack growth simulations with the GFEM ${ }^{\mathrm{gl}}$ are more computationally efficient than with the $h p$-GFEM. Table 1 also shows that the local problems in the GFEM ${ }^{\mathrm{gl}}$ with Strategy 2 are smaller than in the case of Strategy 1. However, Strategy 1 leads to enriched global problems of much smaller size than in the case of Strategy 2. Thus, the algorithm presented in Appendix C is more suitable for Strategy 1 than Strategy 2. A more elaborated cost analysis is performed in Section 4.3.

Table 1 Range of problem sizes solved with GFEM ${ }^{\mathrm{gl}}$ and $h p$-GFEM

\begin{tabular}{lcc}
\hline Method & Min. number of dofs & Max. number of dofs \\
\hline$h p$-GFEM & 31272 & 39042 \\
GFEM $^{\text {gl }}$ with Strategy 1 (global prob.) & 17448 & 17544 \\
GFEM $^{\text {gl }}$ with Strategy 1 (local prob.) & 20040 & 25272 \\
GFEM $^{\text {gl }}$ with Strategy 2 (global prob.) & 18636 & 19836 \\
GFEM $^{\text {gl }}$ with Strategy 2 (local prob.) & 15324 & 23988 \\
\hline
\end{tabular}

\subsection{GFEM $^{\mathrm{gl}}$ Performance in a Mixed-Mode Crack Growth Simulation}

This example considers crack growth of a single edge notched (SEN) specimen in a three point bending configuration. Figure 12 illustrates the global mesh, boundary conditions and the dimensions of the model. The crack is located at the middle of the span and is inclined by an angle $\beta=45^{\circ}$ with respect to the thickness of the beam, which causes a mixed-mode behavior. The geometrical and material parameters of the SEN specimen are as follows: total length, $L_{t}=260 \mathrm{~mm}$, distance between supports, $L_{s}=240 \mathrm{~mm}$, thickness, $t=10 \mathrm{~mm}$, width, $h=60 \mathrm{~mm}$, and ratio of initial crack length to thickness, $a_{o} / h=1 / 3$, Young's modulus $E=2.1 \times 10^{5} \mathrm{~N} / \mathrm{mm}^{2}$ and Poisson's ratio $v=0.3$. The model is subjected to a cyclic load with constant amplitude applied at the middle of the span. The Paris' equation parameters are $C=1.546 \times 10^{-12}\left(\mathrm{~N} / \mathrm{mm}^{2}\right)^{-2.1} \mathrm{~mm}^{-0.05} /$ cycle and $m=2.1$. The cyclic load parameters are $\sigma_{\max }=100 \mathrm{~N} / \mathrm{mm}^{2}$ and $R=0$. The crack growth simulation is performed with 25 incremental crack steps of variable magnitude. The maximum increment along the crack front is taken as $\Delta a_{\max }=0.05 a_{o}$ for the first 4 steps of the simulations and $\Delta a_{\max }=0.075 a_{o}$ for the remainder of the simulations. Details on the computation of crack front increments based on $\Delta a_{\max }$ and Paris' law are provided in, e.g., Section 4.2 of [48].

The inclination of the crack surface with respect to the $z$-axis produces a mixed-mode behavior. In this case, the crack surface tends to rotate such that the angle $\beta$ shown in Figure 12 becomes zero while keeping the middle to the crack front aligned with the center of the applied load area. The main purpose of this example is to verify the performance of the GFEM $^{\mathrm{gl}}$ for crack growth simulations under mixed-mode conditions. The SEN model is solved with both the GFEM ${ }^{\mathrm{gl}}$ and the $h p$-GFEM presented in [48]. Hp-GFEM results are used as a reference. Equivalent discretizations are used in the GFEM ${ }^{\mathrm{gl}}$ and the $h p$-GFEM. Hp-GFEM discretizations use polynomial order $p=3$ and localized refinement with $3.91 \times 10^{-3} \leq L_{e} / a_{o} \leq 1.19 \times 10^{-2}$ along the crack front, where $L_{e}$ is the tetrahedral element size. The localized mesh refinement follows the evolution of the crack front using the refinement and unrefinement technique presented in [48]. Figure 13(a) illustrates the crack surface evolution and the $h p$-GFEM mesh used at various simulation steps. In the case of the GFEM $^{\mathrm{gl}}$, the local problem at each crack step is solved with an $h p$-GFEM discretization with polynomial order $p=3$ and localized refinement with $3.91 \times 10^{-3} \leq L_{e} / a_{o} \leq 1.20 \times 10^{-2}$ along the crack front. Figure 13(b) illustrates the crack surface evolution and the global mesh for the crack growth simulation with the $\mathrm{GFEM}^{\mathrm{gl}}$. It is clear from the figure that the the global mesh remains unchanged throughout the entire crack 


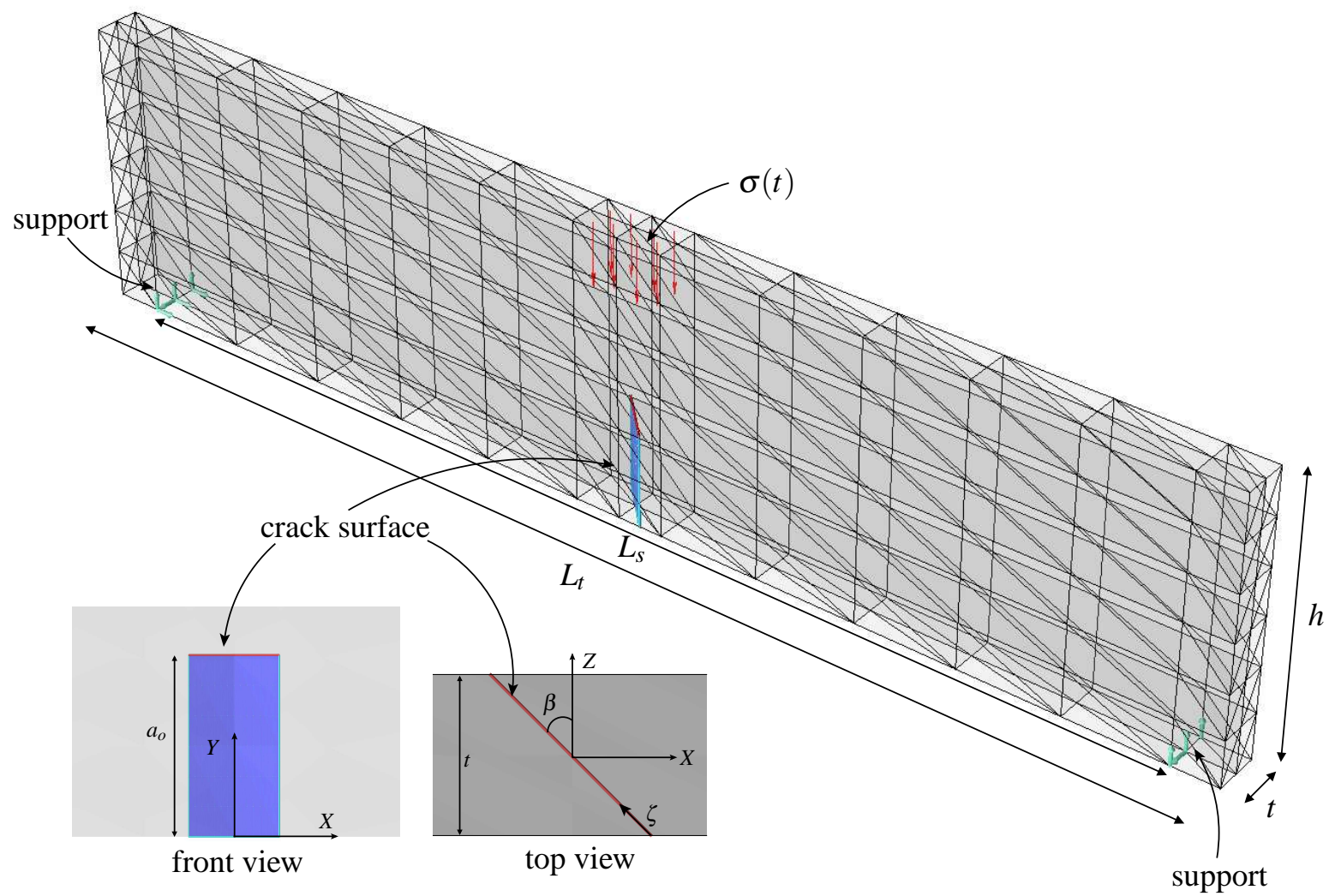

Fig. 12 Single edge notched (SEN) specimen in a three point bending configuration: domain dimensions, crack surface description, and global mesh used with the GFEM ${ }^{\mathrm{gl}}$

growth simulation.

Figure 14 illustrates the $h p$-GFEM and GFEM ${ }^{\mathrm{gl}}$ solutions at crack growth step 25 . In both cases, the solution is plotted in the deformed configuration with an amplification factor. The elements used in the numerical integration of discontinuous functions in $h p$-GFEM and GFEM ${ }^{\mathrm{gl}}$ analyses are used as graphical elements to visualize the discontinuity along the crack surface. The crack surface can be quite complex inside a single computational element, especially in the case of the $\mathrm{GFEM}^{\mathrm{gl}}$ where the global mesh is coarse. Such a feature is allowed by the decoupling between crack surface representation and computational mesh together with the procedure for non-planar cutting of computational elements presented in [46].

Figure 15(a) illustrates the variation of the strain energy with respect to the accumulated maximum crack increment throughout the simulation. One can observe that the strain energies of the solutions computed with GFEM $^{\mathrm{gl}}$ and $h p$-GFEM show very good agreement. Figures 15(b), 15(c), 15(d), 15(e), and 15(f) show the variation of stress intensity factors throughout the simulation at the first, second, middle, second to last and last vertices along the crack front, respectively. The orientation of these vertices is given by the parametric coordinate $\zeta$ illustrated in Figure 12. These vertices are used in the triangularization of the crack surface [46]. The results show that the SIFs computed with GFEM $^{\mathrm{gl}}$ are in good agreement with the corresponding $h p$-GFEM values. The figures also show that both methods can capture very well the transition from a mixed-mode problem at the start of the simulation to a mode- $I$ problem as the crack surface grows and twists around the $y$-axis. 


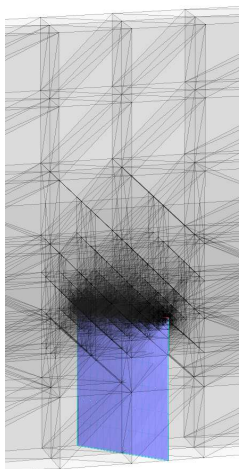

step 0

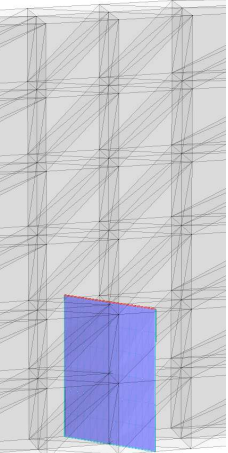

step 0

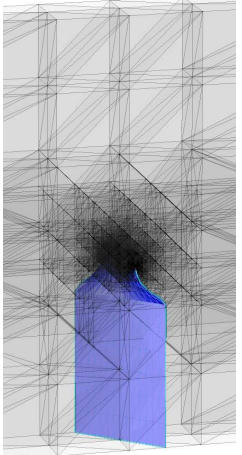

step 5

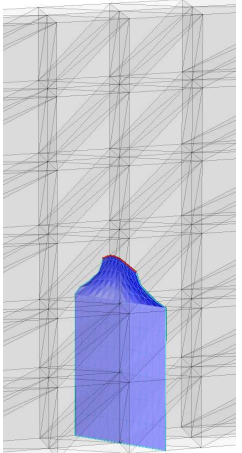

step 5

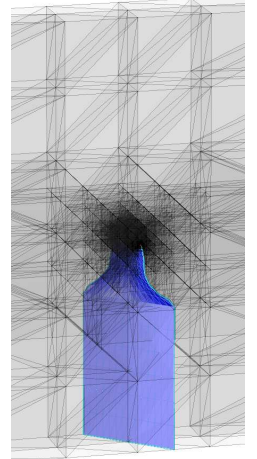

step 10

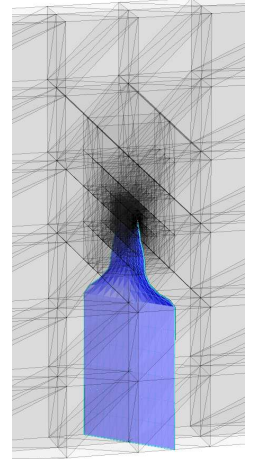

step 15

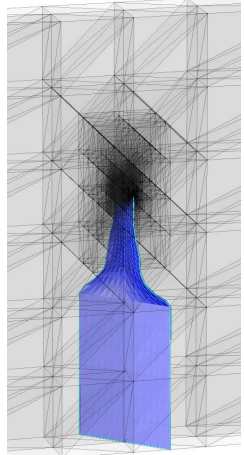

step 20

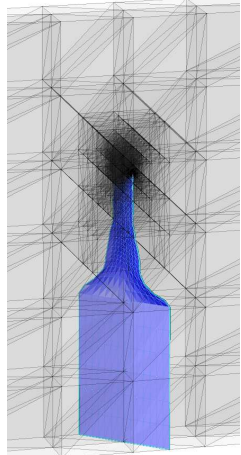

step 25

(a) $h p$-GFEM discretizations

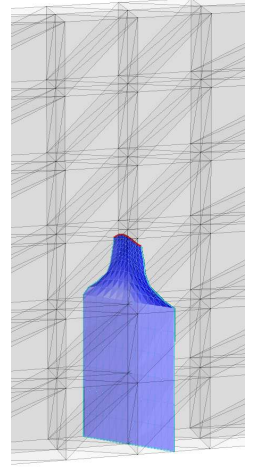

step 10

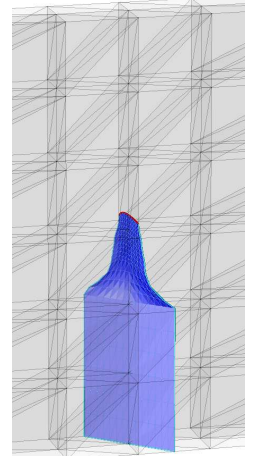

step 15

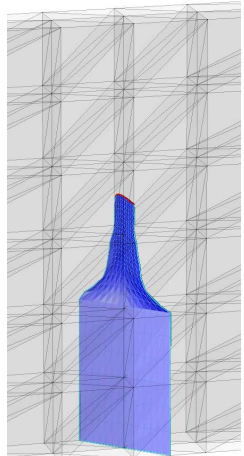

step 20

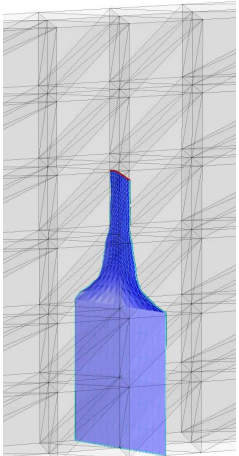

step 25

(b) GFEM $^{\mathrm{gl}}$ global mesh

Fig. 13 Crack surface evolution and mesh for various crack steps in $h p$-GFEM and GFEM ${ }^{\mathrm{gl}}$ simulations 


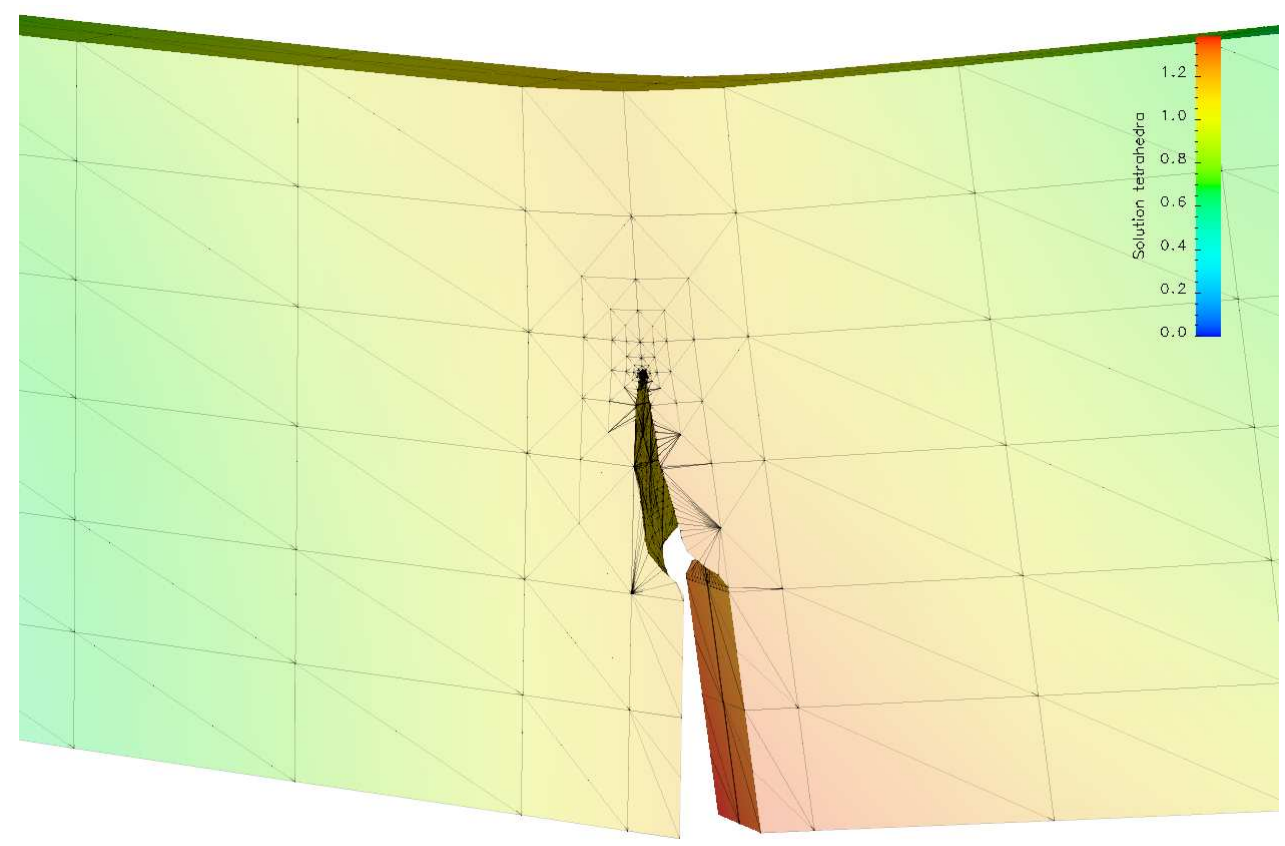

(a) $h p$-GFEM solution

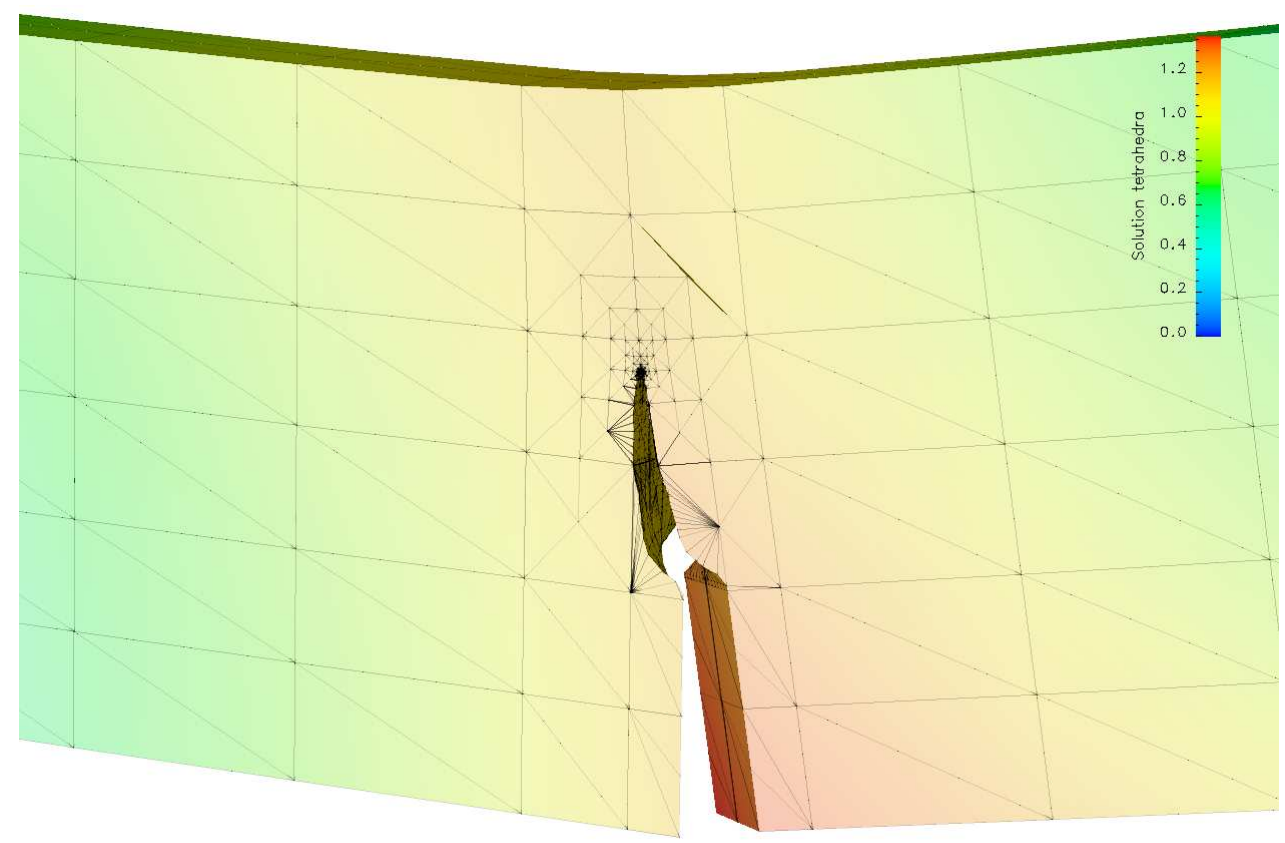

(b) GFEM $^{\mathrm{gl}}$ solution

Fig. $14 h p$-GFEM and GFEM ${ }^{\mathrm{gl}}$ solutions and meshes used in the numerical integration of the weak form shown in the deformed configuration at crack growth step 25 


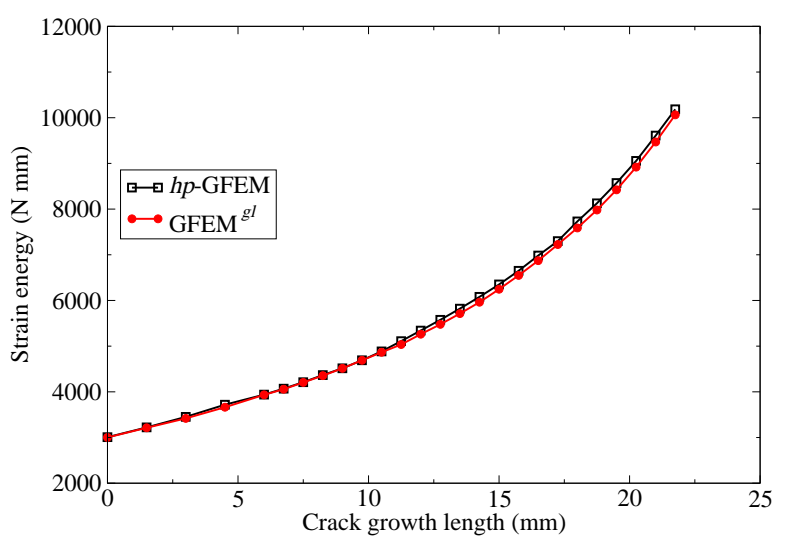

(a) Strain energy

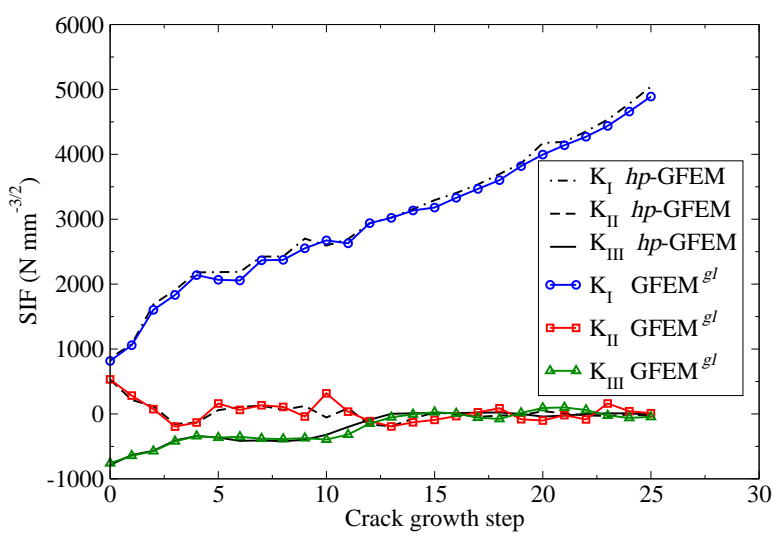

(c) SIFs at second crack front vertex

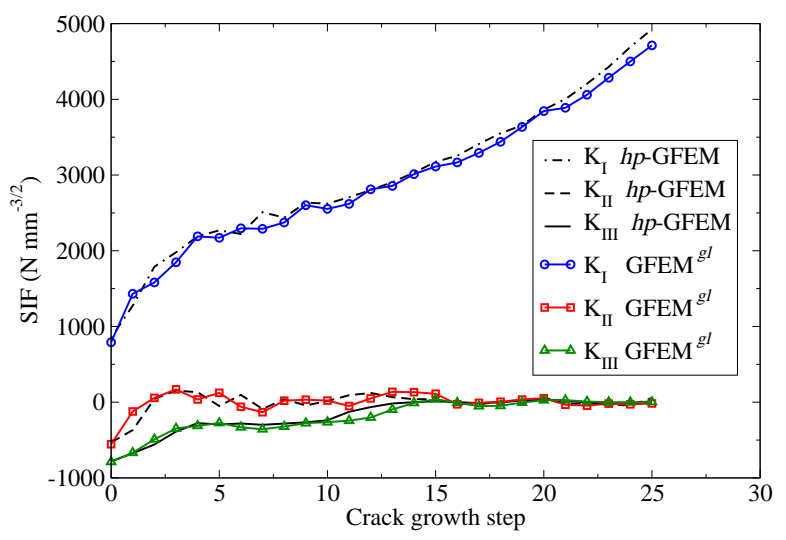

(e) SIFs at second to last crack front vertex

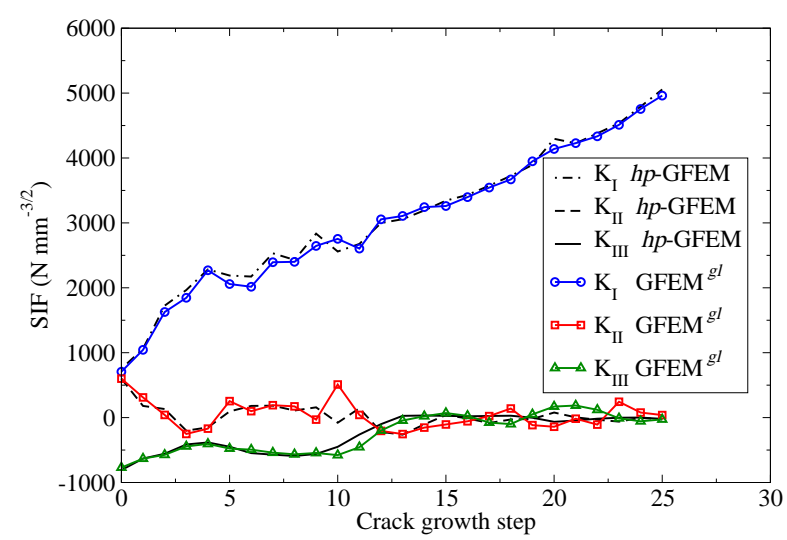

(b) SIFs at first crack front vertex

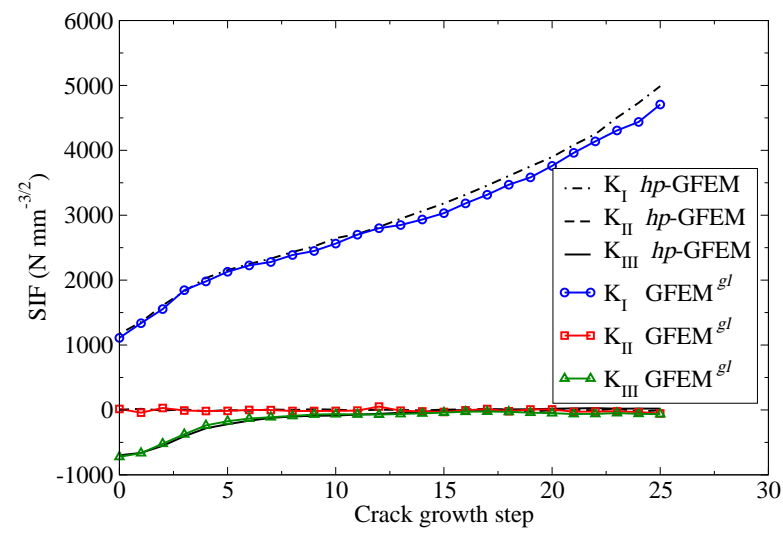

(d) SIFs at middle crack front vertex

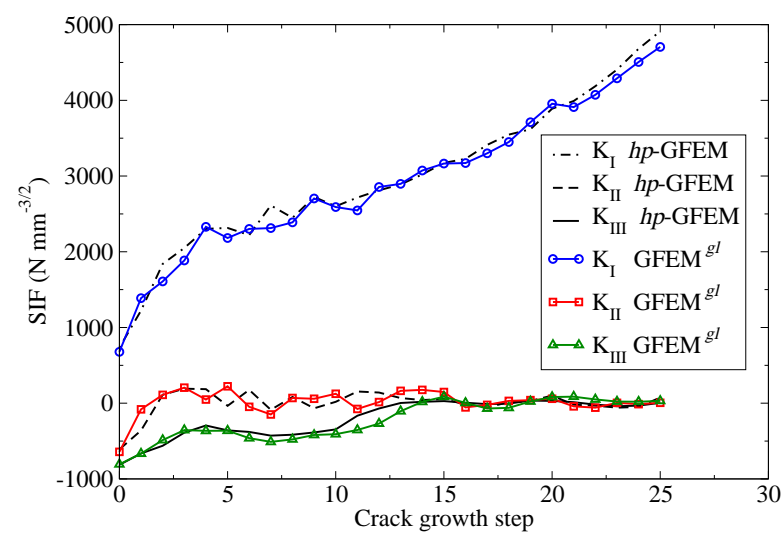

(f) SIFs at last crack front vertex

Fig. 15 Strain energy evolution with respect to crack growth length and stress intensity factors (SIFs) evolution with respect to crack growth step 


\subsection{Computational Cost Analysis of Crack Growth in a 3-D Bracket}

In this section, a three-dimensional bracket model with a half penny-shaped crack is considered. Due to the large number of nodes and elements used in the discretization and the complexity of the geometry, this problem is considerably more challenging for available computational methods than the previous ones. The model illustrated in Figure 16 is subjected to a cyclic load with $R=0$. The load is applied at the horizontal opening and the model is fixed at the vertical openings, as indicated in Figure 16. Figure 16 also illustrates the location and geometry of the initial half penny-shaped crack surface. The radius of the initial crack is $a_{o}=8 \mathrm{~mm}$. Young's modulus and Poisson's ratio of the material are $E=10^{5} \mathrm{MPa}$ and $v=0.33$, respectively. $C=1.425 \times 10^{-11}\left(\mathrm{~N} / \mathrm{mm}^{2}\right)^{-2.5} \mathrm{~mm}^{-0.25} /$ cycle and $m=2.5$ are the parameters of the Paris' equation used in the fatigue model. The simulation has a total of $n=40$ steps, and the maximum values for the crack growth increments used are $\Delta a_{\max }=0.1 a_{o}$ and $\Delta a_{\max }=0.05 a_{o}$ for the initial four steps and the remainder of the simulation, respectively.

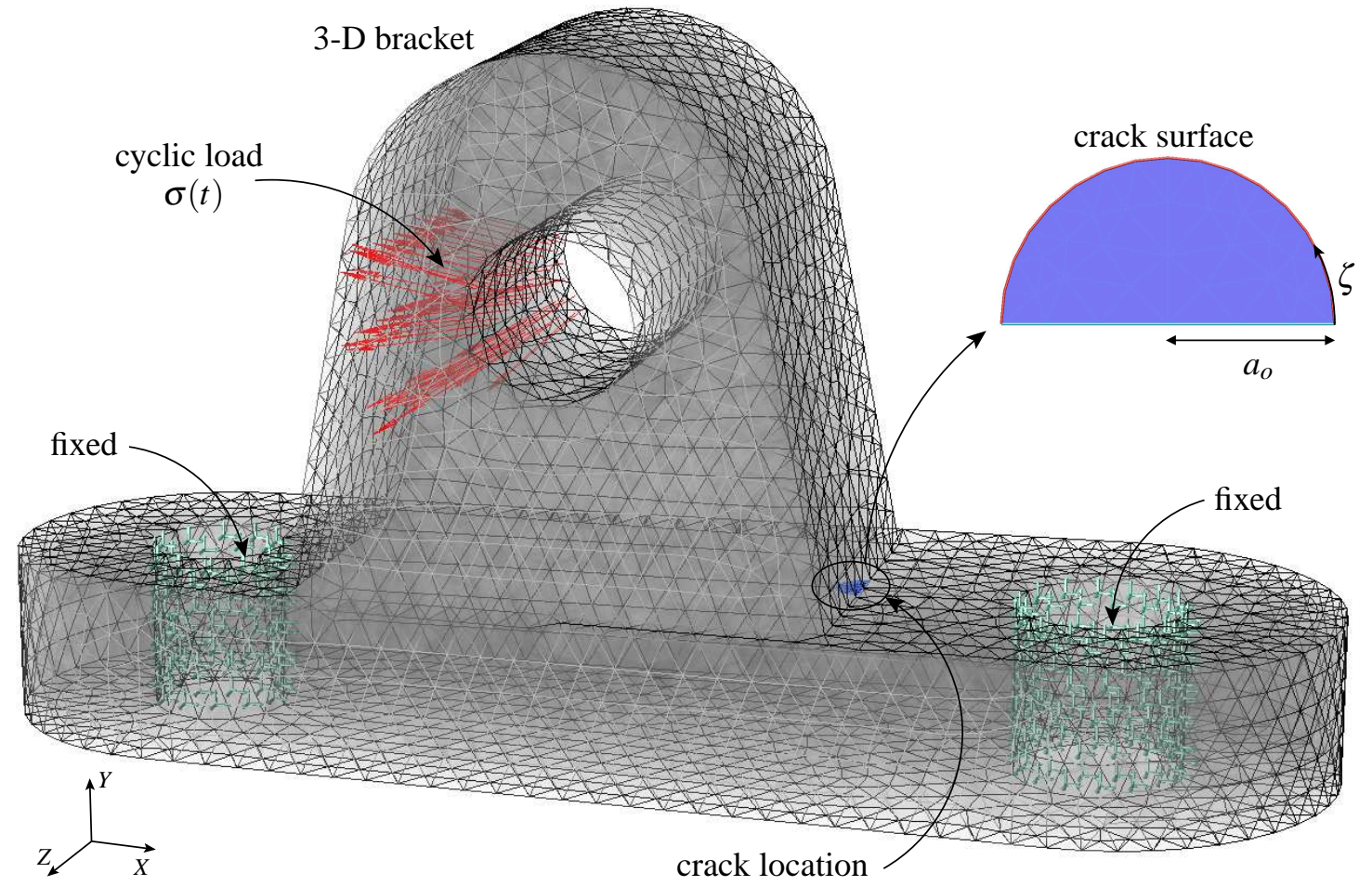

Fig. 16 Three-dimensional bracket model with a half penny-shaped crack

The main goal of this example is to compare the computational performances of the GFEM $^{\mathrm{gl}}$ and the $h p$ GFEM when solving large 3-D problems. As in the previous examples, the $h p$-GFEM solution is used as a reference. Figure 17 illustrates the $h p$-GFEM and GFEM $^{\mathrm{gl}}$ discretizations at crack growth step 20.

In the $h p$-GFEM for fatigue crack growth, as in the standard FEM, a linear system of equations is solved from scratch at each crack growth step. This results in a high computational cost when solving problems with an industrial level of complexity. In contrast, in the $\mathrm{GFEM}^{\mathrm{gl}}$, the solution vector of the initial global problem and the factorization of the initial global stiffness matrix are reused at each crack growth step. This feature of the GFEM $^{\mathrm{gl}}$ leads, as demonstrated below, to crack growth simulations with much reduced computational costs.

In this example, the GFEM $^{\mathrm{gl}}$ strategy shown in Figure 6 together with the Improvement at Starting Step (ISS) discussed in Section 3.5 are used. The local problem domain is created using global elements that intersect the crack surface and their neighbors. As the crack surface evolves, an automatic procedure selects 
and updates the global elements used in the definition of the local problem domain. Once the local problem domain is defined, the local solution is computed using the $h p$-GFEM with polynomial order $p=3$ and localized crack front refinement in the range $1.95 \times 10^{-2} \leq L_{e} / a \leq 1.87 \times 10^{-1}$. Figure 18 illustrates the local problem domains and their solutions at steps $0,8,13,23,32$, and 38 . As in the previous examples, the polynomial order and level of refinement used in $h p$-GFEM discretizations are the same as those adopted in the local problems of the GFEM ${ }^{\mathrm{gl}}$. Figure 19 shows the $h p$-GFEM solutions at crack growth steps $0,8,13$, 23,32 , and 38 .

The accumulated computational cost of the $\mathrm{GFEM}^{\mathrm{gl}}$ and $h p$-GFEM at each crack growth step is presented in Figure 20. In the $h p$-GFEM, the computational cost at each crack step consists of the CPU time for the factorization of the global stiffness matrix, back and forward substitutions. The computational cost of a crack growth step using the GFEM $^{\mathrm{gl}}$ consists of the summation of the CPU time spent on (i) the factorization, back and forward substitutions of the global stiffness matrix of the initial (uncracked) global problem; (ii) factorization, back and forward substitutions of the local stiffness matrix; (iii) computation of the solution of the enriched global problem using the algorithm described in Appendix C; and (iv) Improvement at Starting Step (ISS) described in Section 3.5. In both methods, the factorizations are performed using a parallel sparse solver on a computer with eight cores. The results show that the GFEM $^{\mathrm{gl}}$ for crack growth uses less than half the CPU time required by the $h p$-GFEM.

Tables 2 and 3 list the CPU time and the accumulated computational cost at some representative steps of the crack growth simulation using the $h p$-GFEM and GFEM ${ }^{\mathrm{gl}}$, respectively. They also list the number of dofs used by each method. The results show that the size of the global problem in the GFEM ${ }^{\mathrm{gl}}$ does not depend on the number of dofs used in the local problem. In the GFEM ${ }^{\mathrm{gl}}$, only a small number of dofs are

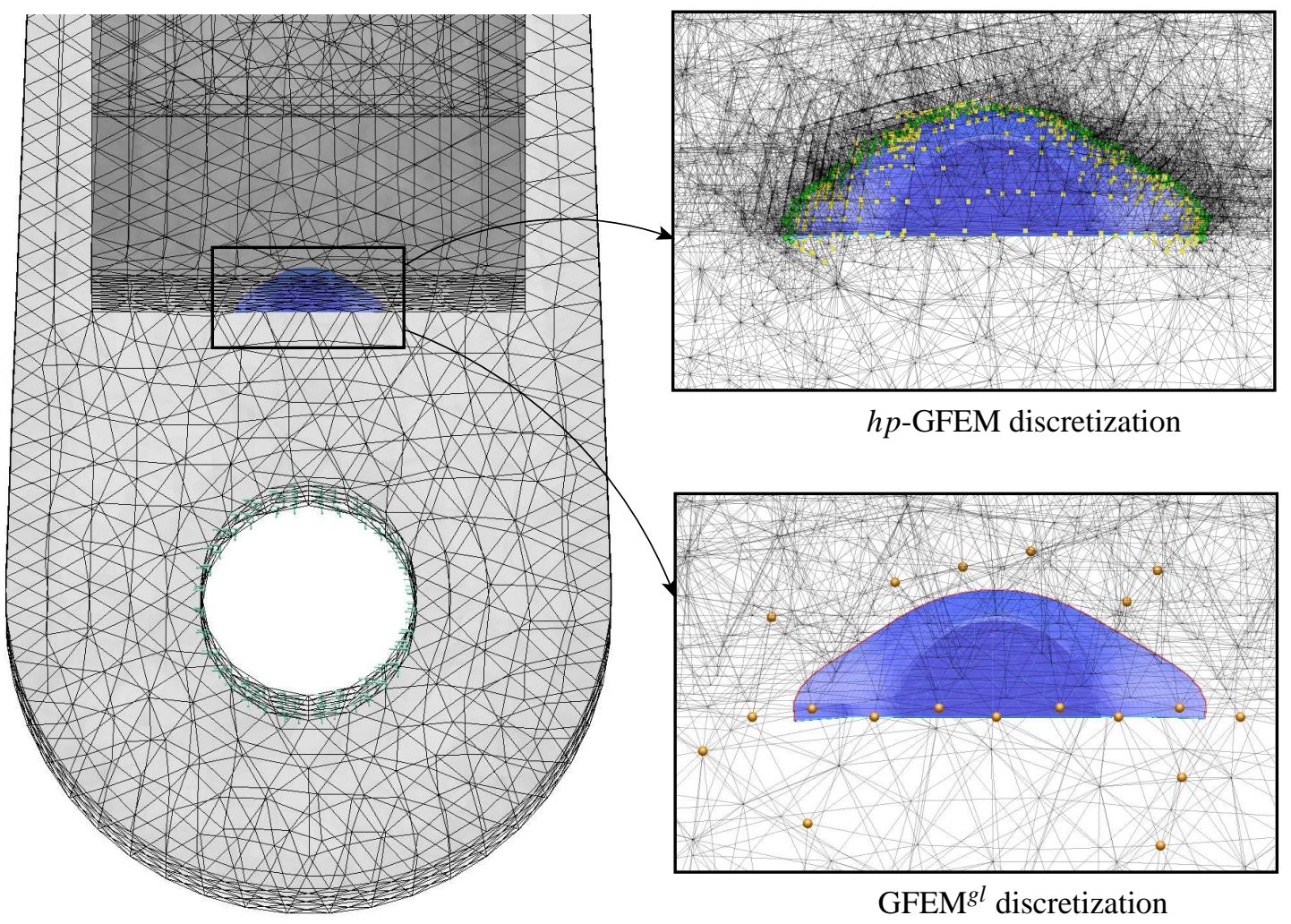

Fig. 17 Crack discretization with $h p$-GFEM and GFEM ${ }^{\mathrm{gl}}$ at crack growth step 20. Yellow cubes, green diamonds, and brown spheres indicate high-order discontinuous, singular, and global-local enrichment functions, respectively. The first two types of enrichments are used in the $h p$-GFEM while the last one is used in the GFEM ${ }^{\mathrm{gl}}$ 


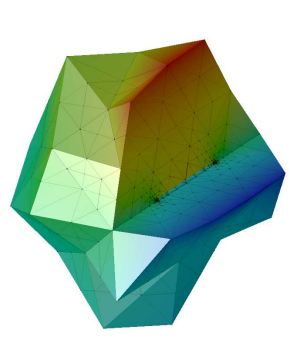

step 0

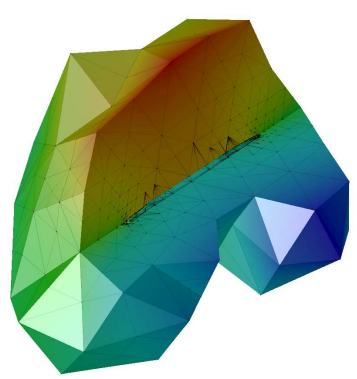

step 23
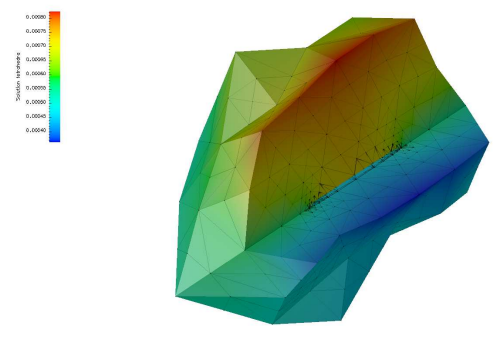

step 8
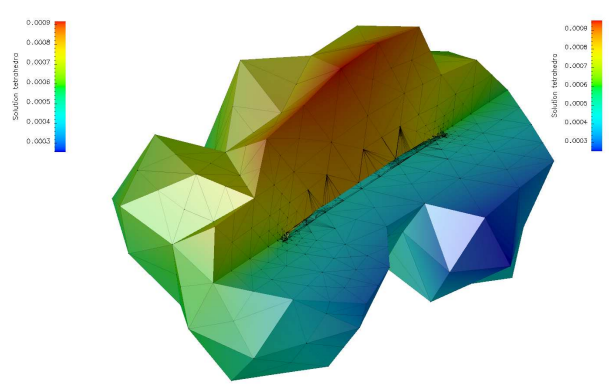

step 32

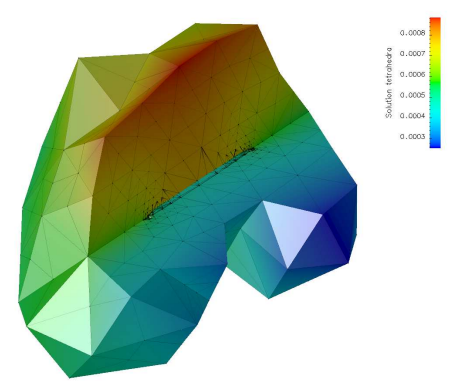

step 13

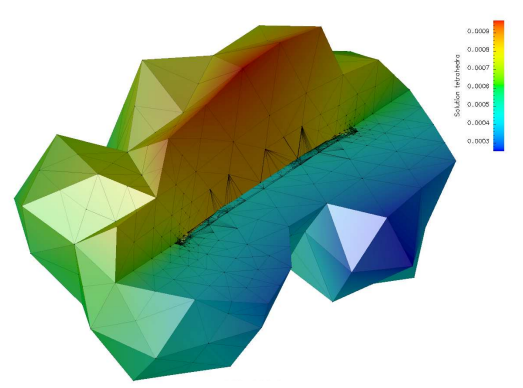

step 38

Fig. 18 Local problem domains and solutions at various crack growth steps. The local domains are automatically selected such that they contain the entire crack surface

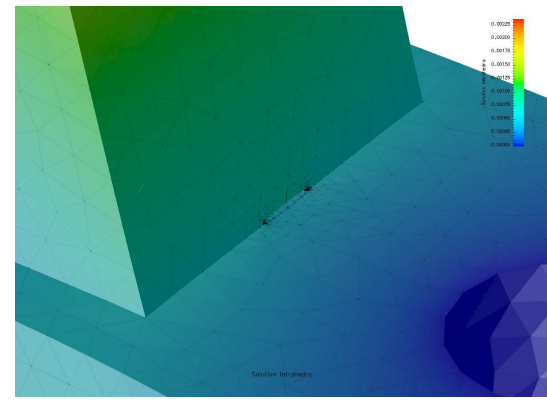

step 0

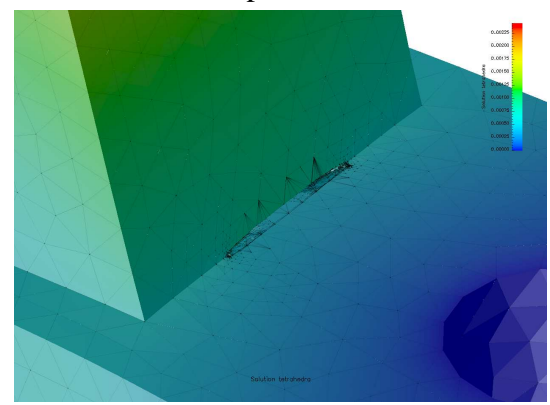

step 23

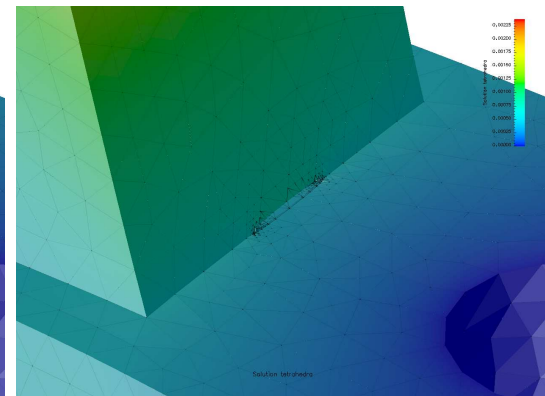

step 8

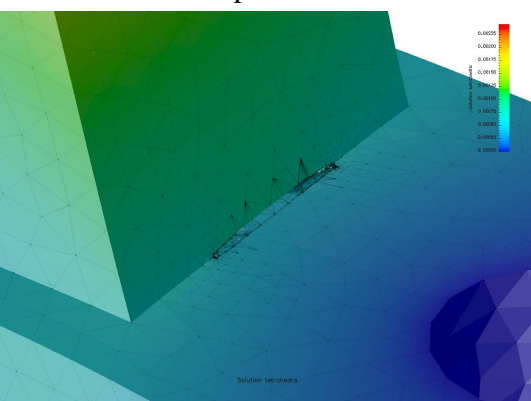

step 32

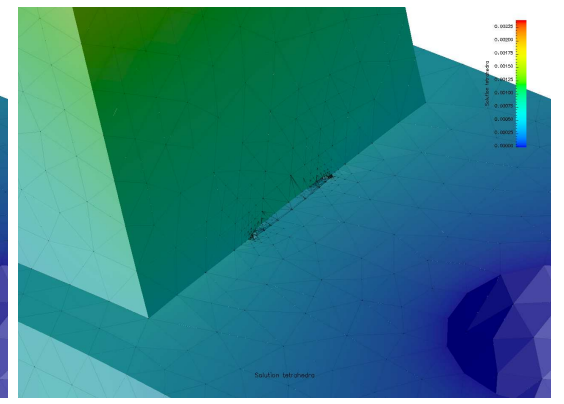

step 13

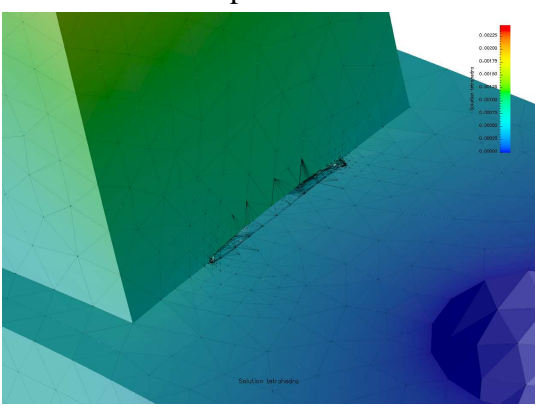

step 38

Fig. $19 h p$-GFEM solutions at various crack growth steps 


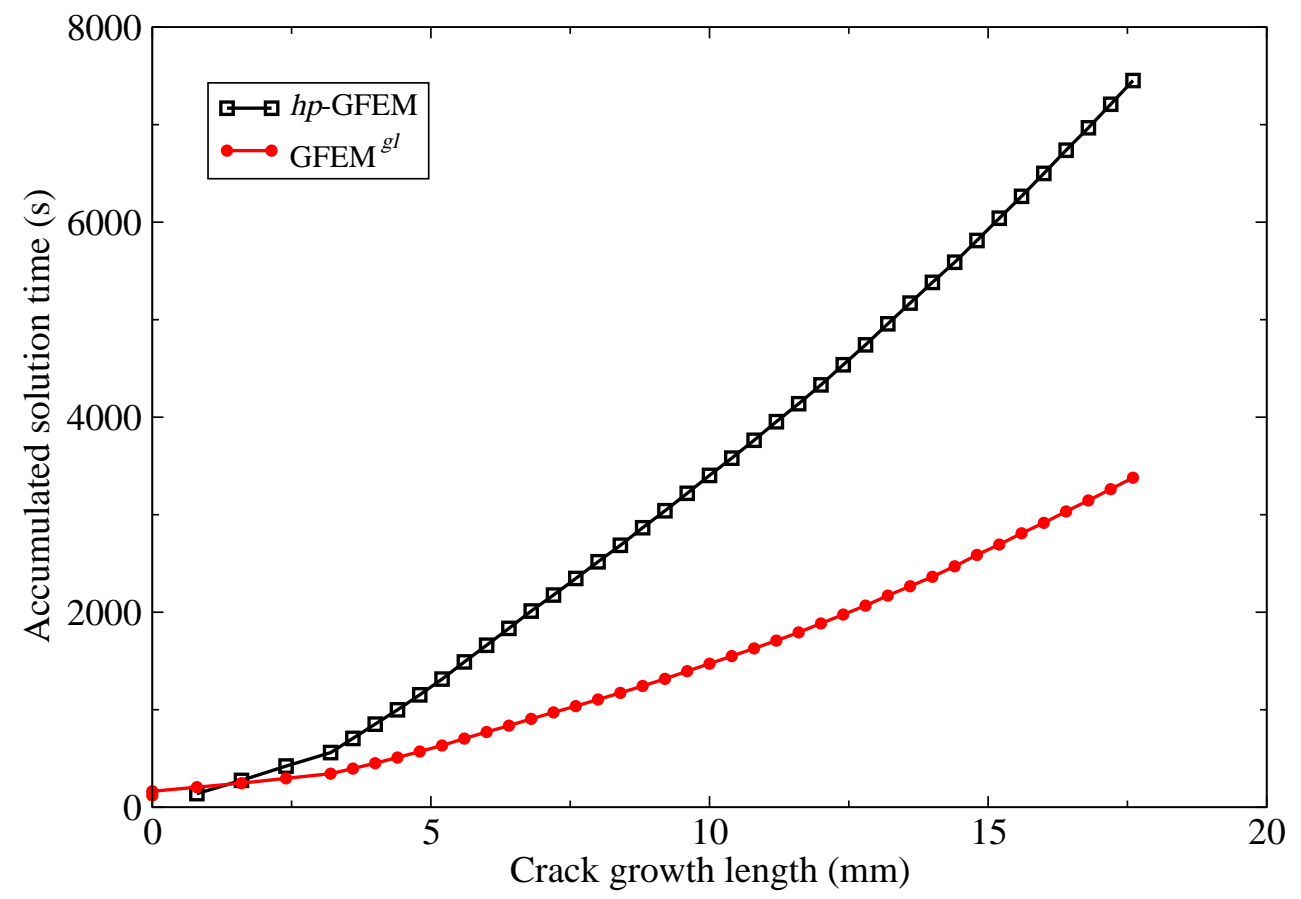

Fig. 20 Accumulated computational cost vs. crack growth length for entire simulation

added to the enriched global problem, e.g., 27, 57, and 84 at crack steps 0,20 , and 35, respectively. In addition, from Table 3, one can observe that the minimum and maximum numbers of global nodes enriched with local solutions are 9 and 28, respectively. In contrast, the localized mesh refinement required by the $h p$-GFEM increases the size of the global problem substantially as the crack propagates. Furthermore, the cost to compute global-local enrichments and to solve the enriched global problem in the GFEM ${ }^{\mathrm{gl}}$ using the algorithm of Appendix C corresponds to between 30\% and 48\% of the CPU time spent with $h p$-GFEM at the same crack step. As a result, the total CPU time spent in the crack growth simulation with the GFEM ${ }^{\mathrm{gl}}$ is much smaller than with the $h p$-GFEM.

Table 2 CPU time spent on the factorization of the stiffness matrix of selected crack steps using the $h p$-GFEM

\begin{tabular}{rccr}
\hline & & & Accumulated \\
Step & Number of degrees of freedom & CPU time(s) & Comp. Cost(s) \\
\hline 0 & 186666 & 139.2 & 139.2 \\
5 & 191388 & 146.3 & 852.2 \\
10 & 204036 & 171.3 & 1661.4 \\
15 & 209892 & 171.2 & 2517.4 \\
20 & 223644 & 183.4 & 3401.0 \\
25 & 230892 & 193.1 & 4331.4 \\
30 & 234282 & 212.0 & 5382.5 \\
35 & 253050 & 234.7 & 6500.0 \\
39 & 255618 & 243.0 & 7452.5 \\
\hline
\end{tabular}

Figures 21(a), 21(b), and 21(c) show the stress intensity factors (SIFs) along the crack front at crack propagation steps 0,10 , and 20, respectively, for $h p$-GFEM and GFEM $^{\mathrm{gl}}$. The SIFs computed with the GFEM ${ }^{\mathrm{gl}}$ 
Table 3 CPU time spent on the factorization of the initial and local problems and on the solution of the enriched global problem of the GFEM ${ }^{\mathrm{gl}}$

\begin{tabular}{|c|c|c|c|c|c|c|c|}
\hline \multirow[b]{2}{*}{ Step } & \multicolumn{3}{|c|}{ Number of dofs } & \multicolumn{3}{|c|}{ CPU time(s) } & \multirow{2}{*}{$\begin{array}{l}\text { Accumulated } \\
\text { Comp. Cost(s) }\end{array}$} \\
\hline & Initial & Local & Enriched & Initial & Local & Enriched & \\
\hline 0 & & 66456 & 115497 & & 33.7 & 8.0 & 205.5 \\
\hline 5 & & 72000 & 115515 & & 40.4 & 13.8 & 451.4 \\
\hline 10 & & 84552 & 115521 & & 52.1 & 15.3 & 771.4 \\
\hline 15 & & 88680 & 115527 & & 51.9 & 16.7 & 1104.7 \\
\hline 20 & 115470 & 95532 & 115527 & 77.3 & 59.9 & 16.7 & 1472.5 \\
\hline 25 & & 112536 & 115530 & & 73.4 & 18.2 & 1885.0 \\
\hline 30 & & 115374 & 115551 & & 74.3 & 23.8 & 2363.9 \\
\hline 35 & & 124992 & 115554 & & 82.2 & 25.1 & 2916.5 \\
\hline 39 & & 134646 & 115554 & & 91.9 & 25.2 & 3379.1 \\
\hline
\end{tabular}

show good agreement with the reference values extracted from the $h p$-GFEM solution. Figure 21(d) shows the variation of the strain energy of the quasi-static solution as a function of crack growth length. Once more, GFEM ${ }^{\mathrm{gl}}$ and $h p$-GFEM solutions show good agreement.

Figures 22(a) and 22(b) illustrate the crack surface evolution computed with the $h p$-GFEM and the GFEM ${ }^{\mathrm{gl}}$, respectively. The crack surfaces are presented at steps 5, 15, 25, and 35. One can observe that both methods lead to nearly identical crack surface predictions. Both methods capture the three-dimensional mixed-mode behavior of the solution and provide non-planar crack surface predictions.

\section{Summary and Conclusions}

This paper presents a GFEM with enrichment functions built on-the-fly through a global-local analysis. The methodology is applied to high-cycle fatigue crack growth in three-dimensional bodies. Boundary conditions for the fine-scale problem containing the crack surface at a propagation step are provided by the coarse-scale solution computed at the previous step, and thus they are dynamically updated as the crack grows. This is, to our knowledge, a unique methodology to build approximation spaces for this class of problems. The fine-scale solutions are embedded in the coarse-scale solution spaces using the partition of unity method $[4,3,34,14,15,16]$.

The proposed global-local enrichments add only three degrees of freedom to nodes of the coarse-scale discretization. In the example solved in Section 4.3, the uncracked coarse-scale discretization has 115470 dofs. The global-local enrichments used to model a complex 3-D crack surface adds at most 84 dofs to the problem. In contrast, if available methods like the FEM with remeshing are used, the discretization of 3-D crack surfaces requires a large number of degrees of freedom (cf. Table 2).

The coarse-scale mesh used in the GFEM $^{\text {gl }}$ need not model the crack surface explicitly. Instead, the cracks are modeled through global-local enrichment functions - the solution of fine-scale problems computed with the $h p$-GFEM presented in [46, 47]. As a result, the coarse-scale mesh remains unchanged during the simulation. This, combined with the hierarchical nature of GFEM shape functions, allows the recycling of the factorization of the global stiffness matrix during a crack growth simulation. An algorithm exploring this feature of the method is presented in Appendix C. The numerical example presented in Section 4.3 shows that the computational cost to solve the problem at each crack growth step is much smaller than in available methods. The computational cost of the GFEM $^{\mathrm{gl}}$ can be further reduced. It is clear from Table 3 


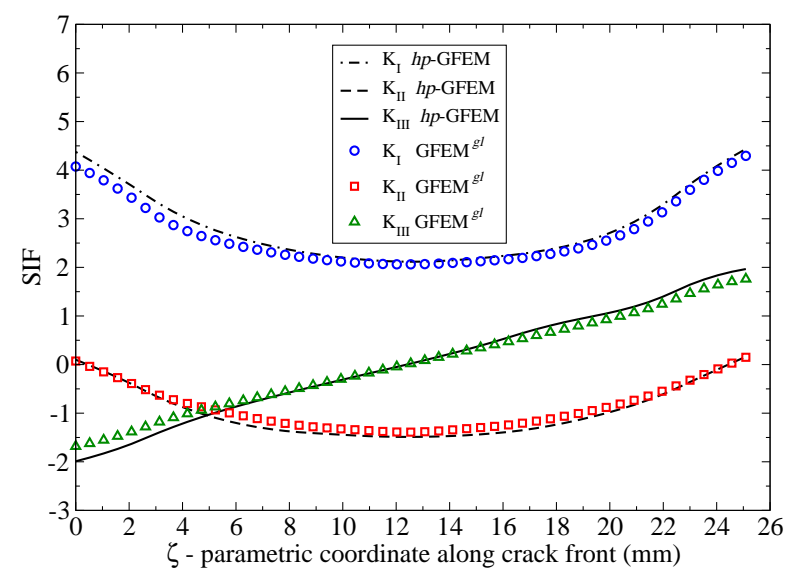

(a) SIFs along crack front for step 0

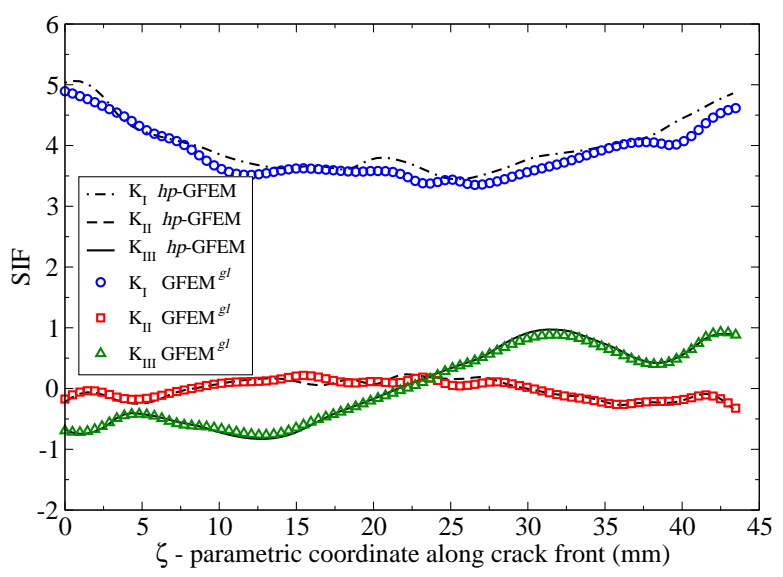

(c) SIFs along crack front for step 20

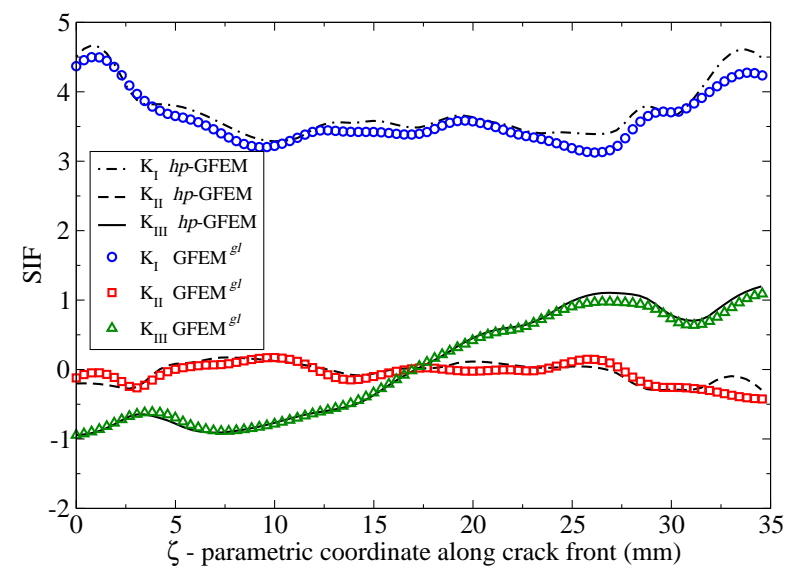

(b) SIFs along crack front for step 10

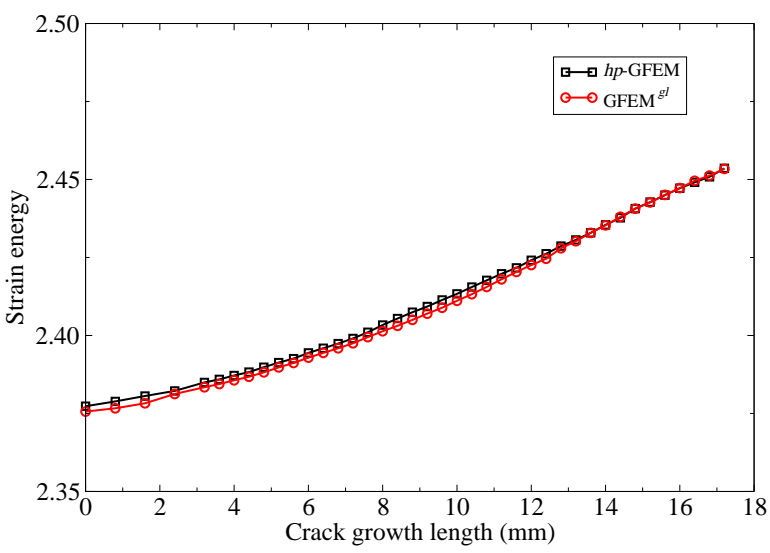

(d) Strain energy with respect to the crack growth length

Fig. 21 Stress intensity factors (SIFs) at various steps and strain energy variation throughout the simulation

that the cost to solve the coarse-scale problem in the GFEM ${ }^{\mathrm{gl}}$ is dominated by the cost of the local problem solution. In this paper, a single local problem is defined at each crack propagation step. However, a local problem can be defined for each node of the coarse-scale mesh whose partition of unity support intersects the crack surface. These local problems can be efficiently solved in parallel, since no communication among processors solving different local problems is required [31]. This leads to very scalable computations even on shared memory machines [31], which is a commodity hardware nowadays.

The computational efficiency of the GFEM ${ }^{\mathrm{gl}}$ does not come at the expense of its accuracy - the computational accuracy of the GFEM $^{\mathrm{gl}}$ is comparable to the $h p$-GFEM [48]. This is demonstrated in Section 4 where 3-D mixed-mode problems with complex crack surfaces are solved. This high accuracy is delivered using global meshes with elements that are orders of magnitude larger than those required by, e.g., the standard FEM. As a result, the crack surface can be quite complex inside a single finite element in the global mesh. Such a feature is allowed by decoupling the crack surface representation from the computational mesh [46]. In summary, this paper demonstrates that the GFEM $^{\mathrm{gl}}$ is a fast and reliable alternative for the simulation of mixed-mode crack growth problems in complex 3-D domains.

Acknowledgments: The support from the U.S. Air Force Office of Scientific Research under contract number FA9550-09-1-0401 and the U.S. Air Force Research Laboratory Air Vehicles Directorate under contract number USAF-0060-50-0001 is gratefully acknowledged. 


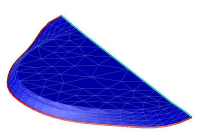

step 5

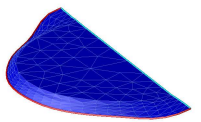

step 5

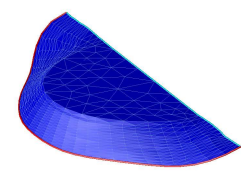

step 15

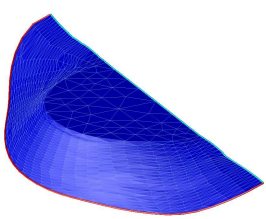

step 25

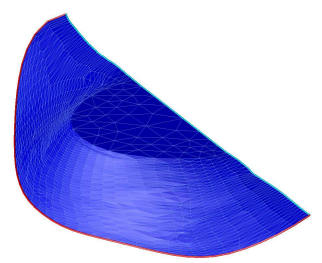

step 35

(a) Crack surface evolution computed with $h p$-GFEM

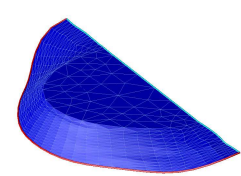

step 15

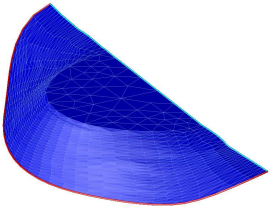

step 25

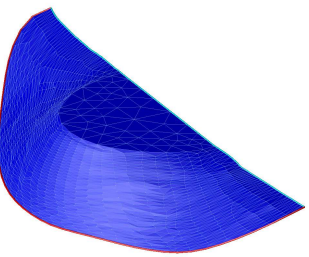

step 35

(b) Crack surface evolution computed with GFEM $^{\text {gl }}$

Fig. 22 Crack surface at various propagation steps

\section{A Update of Crack Surface}

In step (2d) of the algorithm presented in Section 3.5, the crack surface is updated using the solution of the enriched global problem $\boldsymbol{u}_{G}^{k+1}$. At each crack propagation step, the magnitude and direction of the crack front advance are computed as described below. New position vectors of crack front vertices are computed and the Face Offsetting Method (FOM) [28] is used to avoid self-intersections of non-convex crack fronts. This is the same approach presented in [48] and the reader is referred to Section 2.2 of that paper for further details.

Crack growth direction - Schöllmann's criterion In 3-D mixed-mode crack growth problems, the crack front deflection at each crack step is represented by a kinking angle and a twisting angle as illustrated in Figure 23. In this work, Schöllmann's criterion [53] is adopted. This criterion is equivalent to the criterion of maximum tangential stress proposed by Erdogan and Sih [20] when the mode III stress intensity factor, $K_{I I I}$, is zero.

The crack kinking angle $\theta_{0}$ is a non-linear function of $K_{I}, K_{I I}$, and $K_{I I I}$, the stress intensity factors for modes $I, I I$, and $I I I$, respectively. The reader is referred to [53] or to Section 4.1 of [48] for further details. Once the deflection angle $\theta_{0}$ is determined, the twisting angle $\psi_{0}$ is defined as in [53] by

$$
\psi_{0}=\frac{1}{2} \arctan \left[\frac{2 \tau_{\theta z}\left(\theta_{0}\right)}{\sigma_{\theta}\left(\theta_{0}\right)-\sigma_{z}\left(\theta_{0}\right)}\right]
$$

where $\sigma_{\theta}, \tau_{\theta z}$, and $\sigma_{z}$ are components of the stress tensor in a cylindrical coordinate system defined at the crack front.

Magnitude of crack front advance - Paris-Erdogan equation In this paper, Paris-Erdogan equation [44]

$$
\frac{d a}{d N}=C(\Delta K)^{m}
$$




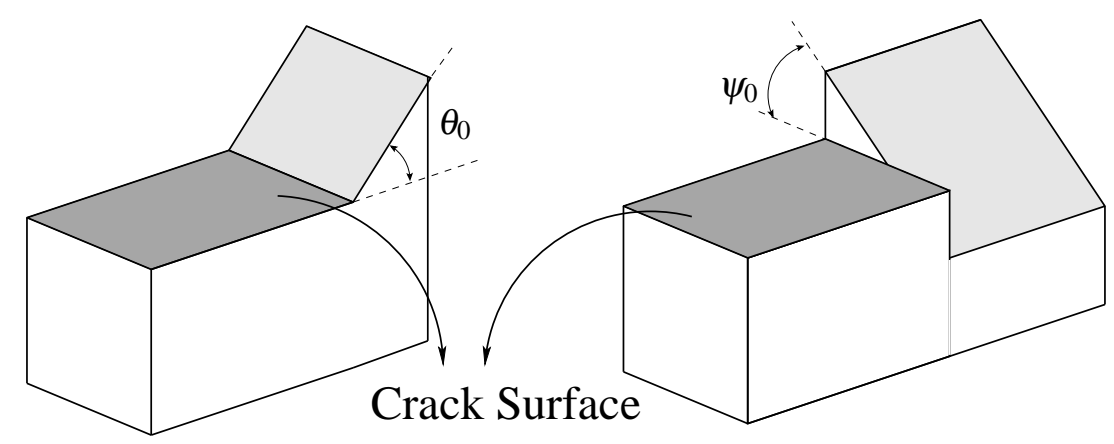

Fig. 23 Crack kinking and twisting angles $\theta_{0}$ and $\psi_{0}$ for three-dimensional mixed-mode crack problems [52]

is used to predict the crack growth rate. Parameters $C$ and $m$ are regarded as material constants, while $\Delta K=(1-R) K_{\max }$ is the stress intensity factor range under cyclic loading, where $R$ is the ratio of minimum to maximum loads applied in a cycle and $K_{\max }$ is the stress intensity factor at the maximum load. In Equation (18), $\Delta K$ takes into account mode $I$ only. Mixed-mode effects are taken into account by using instead the cyclic comparative stress intensity factor, $\Delta K_{v}$, given by [52]

$$
\Delta K_{v}=\frac{\Delta K_{I}}{2}+\frac{1}{2} \sqrt{\Delta K_{I}^{2}+4\left(\alpha_{1} \Delta K_{I I}\right)^{2}+4\left(\alpha_{2} \Delta K_{I I I}\right)^{2}}
$$

where $\alpha_{1}=K_{I c} / K_{I I c}$ and $\alpha_{2}=K_{I c} / K_{I I I c}$ are the ratios of the fracture toughness of mode $I$ to mode $I I$ and of mode $I$ to mode $I I I$, respectively $[53,52]$. In the examples presented in this paper, we adopt $\alpha_{1}=1.155$ and $\alpha_{2}=1.0$ as proposed in [53,52].

The reader is referred to Section 4.2 of [48] for further details on the application of Equation (18) to compute crack front advance and fatigue life.

\section{B Definition of Local Domains}

As illustrated in Figures 6 and 8, local problem domains are defined by either global clouds that intersect the crack surface or by global clouds that intersect the crack front. Let $\mathscr{I}_{S_{k+1}}$ denote the indices of all clouds from the global mesh that intersect the crack surface $S_{k+1}$. These are also called seed nodes and are illustrated with brown spheres in Figure 6. The local domain $\Omega_{L}^{k}$ containing crack surface $S_{k+1}$ (cf. Figure 3 for notation) is given by

$$
\Omega_{L}^{k}=\bigcup_{\beta \in \mathscr{I}_{S_{k+1}}} \omega_{\beta}
$$

where the cloud $\omega_{\beta}$ is the union of (copy of) global elements sharing vertex node $\boldsymbol{x}_{\beta}, \beta \in \mathscr{I}_{S_{k+1}}$.

This can be implemented as follows:

(i) Find all global elements that intersect crack surface $S_{k+1}$; Mark the nodes of these elements as seed nodes.

(ii) The local domain $\Omega_{L}^{k}$ is defined by the union of (copy of) global elements sharing a seed node.

Local domains containing the crack front only are defined analogously:

(i) Find all global elements that intersect the front, $\Gamma_{k+1}$, of the crack surface $S_{k+1}$; Mark the nodes of these elements as seed nodes.

(ii) The local domain $\Omega_{L}^{k}$ is defined by the union of (copy of) global elements sharing a seed node. 
This simple algorithm can be used to create local domains containing fairly complex 3-D crack surfaces (or crack fronts only) like those shown in Figures 13 and 22.

\section{Solution of Enriched Global Problem}

This section presents an algorithm to efficiently solve the system of equations associated with the enriched coarse-scale problem (9). The algorithm is an extension of the procedure presented in Section A.2 of [13] to crack propagation problems. It explores the hierarchical nature of the global-local enrichment functions and reuses in all crack propagation steps the solution and the factorization of the stiffness matrix associated with the initial global problem (7). All quantities presented in this section are global; therefore, the subscript $(.)_{G}$ is hereafter dropped.

The enriched global space $\boldsymbol{X}_{G}^{k}(\Omega)$ defined in (12) is the union of the initial global space $\boldsymbol{X}_{G}^{0}(\Omega)$ and globallocal GFEM shape functions. As a result, the global stiffness matrix of the initial global problem (7), $\boldsymbol{K}^{0}$, is nested in the global matrix of the enriched global problem (9) at any crack propagation step $k$. Thus, the system of equations associated with problem (9) can be partitioned as follows:

$$
\left[\begin{array}{cc}
\boldsymbol{K}^{0} & \boldsymbol{K}^{0, g l(k)} \\
\boldsymbol{K}^{g l(k), 0} & \boldsymbol{K}^{g l(k)}
\end{array}\right]\left[\begin{array}{c}
\underline{\boldsymbol{u}}^{0(k)} \\
\underline{\underline{u}}^{g l(k)}
\end{array}\right]=\left[\begin{array}{c}
\boldsymbol{F}^{0} \\
\boldsymbol{F}^{g l(k)}
\end{array}\right]
$$

where $\boldsymbol{K}^{g l(k)}$ and $\underline{\boldsymbol{u}}^{g l(k)}$ are the global stiffness matrix entries and dofs, respectively, associated with globallocal GFEM shape functions used in the definition of space $\boldsymbol{X}_{G}^{k}(\Omega)$. Vector $\underline{\boldsymbol{u}}^{0(k)}$ has dofs associated with GFEM shape functions from the initial global space $\boldsymbol{X}_{G}^{0}(\Omega)$. From the first equation in (21), it follows that

$$
\boldsymbol{K}^{0} \underline{\boldsymbol{u}}^{0(k)}+\boldsymbol{K}^{0, g l(k)} \underline{\boldsymbol{u}}^{g l(k)}=\boldsymbol{F}^{0} .
$$

Thus

$$
\underline{\boldsymbol{u}}^{0(k)}=\underline{\boldsymbol{u}}^{0}-\boldsymbol{S}^{0, g l(k)} \underline{\boldsymbol{u}}^{g l(k)}
$$

where $\underline{\boldsymbol{u}}^{0}=\left(\boldsymbol{K}^{0}\right)^{-1} \boldsymbol{F}^{0}$ is the solution vector of the initial global problem and matrix $\boldsymbol{S}^{0, g l(k)}$ is the solution of

$$
\boldsymbol{K}^{0} \boldsymbol{S}^{0, g l(k)}=\boldsymbol{K}^{0, g l(k)}
$$

which can be computed at a lower cost using the available factorization of the initial global stiffness matrix, $\left(\boldsymbol{K}^{0}\right)^{-1}$.

From the second equation in (21), it follows that

$$
\boldsymbol{K}^{g l(k), 0} \underline{\boldsymbol{u}}^{0(k)}+\boldsymbol{K}^{g l(k)} \underline{\boldsymbol{u}}^{g l(k)}=\boldsymbol{F}^{g l(k)}
$$

Using Equation (23) to condense out the fine-scale dofs, $\underline{\boldsymbol{u}}^{g l(k)}$, the above reduces to

$$
\hat{\boldsymbol{K}}^{g l(k)} \underline{\underline{u}}^{g l(k)}=\hat{\boldsymbol{F}}^{g l(k)}
$$

where

$$
\hat{\boldsymbol{K}}^{g l(k)}=\boldsymbol{K}^{g l(k)}-\boldsymbol{K}^{g l(k), 0} \boldsymbol{S}^{0, g l(k)}
$$

and

$$
\hat{\boldsymbol{F}}^{g l(k)}=\boldsymbol{F}^{g l(k)}-\boldsymbol{K}^{g l(k), 0} \underline{\boldsymbol{u}}^{0} .
$$

The computation of the solution vector of the enriched global problem,

$$
\underline{\boldsymbol{u}}^{k}=\left[\begin{array}{ll}
\underline{\boldsymbol{u}}^{0(k)} & \underline{\boldsymbol{u}}^{g l(k)}
\end{array}\right]^{T},
$$


involves back and forward substitutions on the factorization of the global stiffness matrix, $\boldsymbol{K}^{0}$, some matrix multiplications, and the solution of the system (26). This procedure leads to significant savings in computational cost when solving large problems, where $\operatorname{dim}\left(\underline{\boldsymbol{u}}^{0(k)}\right) \gg \operatorname{dim}\left(\underline{\boldsymbol{u}}^{g l(k)}\right)$. Thus, the solution of the enriched global problem at each crack growth step can be obtained in a very efficient manner. This is demonstrated numerically in Section 4.3.

\section{References}

[1] Areias P, Belytschko T (2005) Analysis of three-dimensional crack initiation and propagation using the extended finite element method. International Journal for Numerical Methods in Engineering 63:760788

[2] Babuška I, Melenk J (1995) The partition of unity finite element method. Tech. Rep. BN-1185, Inst. for Phys. Sc. and Tech., University of Maryland

[3] Babuška I, Melenk J (1997) The partition of unity method. International Journal for Numerical Methods in Engineering 40:727-758

[4] Babuška I, Caloz G, Osborn J (1994) Special finite element methods for a class of second order elliptic problems with rough coefficients. SIAM Journal on Numerical Analysis 31(4):945-981

[5] Belytschko T, Black T (1999) Elastic crack growth in finite elements with minimal remeshing. International Journal for Numerical Methods in Engineering 45:601-620

[6] Belytschko T, Gracie R, Ventura G (2009) A review of extended/generalized finite element methods for material modeling. Modelling and Simulations in Materials Science and Engineering 17:24pp, http://dx.doi.org/10.1088/0965-0393/17/4/043001

[7] Ben Dhia H, Jamond O (2010) On the use of XFEM within the Arlequin framework for the simulation of crack propagation. Computer Methods in Applied Mechanics and Engineering 199:1403-1414

[8] Bordas S, Moran B (2006) Enriched finite elements and level sets for damage tolerance assessment of complex structures. Engineering Fracture Mechanics 73:1176-1201

[9] Chahine E, Laborde P, Renard Y (2008) Spider-xfem, an extended finite element variant for partially unknown crack-tip displacement. European Journal of Computational Mechanics 15(5-7):625-636

[10] Chahine E, Laborde P, Renard Y (2009) A reduced basis enrichment for the extended finite element method. Mathematical Modelling of Natural Phenomena 4(1):88-105

[11] dell'Erba D, Aliabadi M (2000) Three-dimensional thermo-mechanical fatigue crack growth using BEM. International Journal of Fatigue 22:261-273

[12] Duarte C (1996) The hp Cloud Method. PhD dissertation, The University of Texas at Austin, austin, TX, USA

[13] Duarte C, Kim DJ (2008) Analysis and applications of a generalized finite element method with globallocal enrichment functions. Computer Methods in Applied Mechanics and Engineering 197(6-8):487504, DOI 10.1016/j.cma.2007.08.017

[14] Duarte C, Oden J (1995) Hp clouds-A meshless method to solve boundary-value problems. Tech. Rep. 95-05, TICAM, The University of Texas at Austin 
[15] Duarte C, Oden J (1996) An $h p$ adaptive method using clouds. Computer Methods in Applied Mechanics and Engineering 139:237-262

[16] Duarte C, Oden J (1996) Hp clouds - An $h p$ meshless method. Numerical Methods for Partial Differential Equations 12:673-705

[17] Duarte C, Babuška I, Oden J (2000) Generalized finite element methods for three dimensional structural mechanics problems. Computers and Structures 77:215-232

[18] Duarte C, Hamzeh O, Liszka T, Tworzydlo W (2001) A generalized finite element method for the simulation of three-dimensional dynamic crack propagation. Computer Methods in Applied Mechanics and Engineering 190(15-17):2227-2262, DOI 10.1016/S0045-7825(00)00233-4

[19] Duarte C, Kim DJ, Babuška I (2007) Chapter: A global-local approach for the construction of enrichment functions for the generalized fem and its application to three-dimensional cracks. In: Leitão V, Alves C, Duarte C (eds) Advances in Meshfree Techniques, Springer, The Netherlands, Computational Methods in Applied Sciences, vol 5, iSBN 978-1-4020-6094-6

[20] Erdogan F, Sih G (1963) On the crack extension in plates under plane loading and transverse shear. Journal of Basic Engineering 85:519-525

[21] Fan R, Fish J (2008) The rs-method for material failure simulations. International Journal for Numerical Methods in Engineering 73(11):1607-1623, doi: 10.1002/nme.2134

[22] Fish J, Nath A (1993) Adaptive and hierarchical modelling of fatigue crack propagation. International Journal for Numerical Methods in Engineering 36:2825-2836

[23] Fries TP, Belytschko T (2010) The generalized/extended finite element method: An overview of the method and its applications. International Journal for Numerical Methods in Engineering pp 253-304

[24] Galland F, Gravouil A, Malvesin E, Rochette M (2011) A global model reduction approach for 3D fatigue crack growth with confined plasticity. Computer Methods in Applied Mechanics and Engineering DOI 10.1016/j.cma.2010.08.018

[25] Gravouil A, Moës N, Belytschko T (2002) Non-planar 3d crack growth by the extended finite element and level sets - Part II: Level set update. International Journal for Numerical Methods in Engineering 53(11):2569-2586

[26] Guidault PA, Allix O, Champaney L, Cornuault C (2008) A multiscale extended finite element method for crack propagation. Computer Methods in Applied Mechanics and Engineering 197:381-399

[27] Hou T, Wu XH (1997) A multiscale finite element method for elliptic problems in composite materials and porous media. Journal of Computational Physics 134:169-189

[28] Jiao X (2007) Face offsetting: A unified framework for explicit moving interfaces. Journal of Computational Physics 220(2):612-625

[29] Kim DJ, Duarte C, Proenca S (2009) Generalized finite element method with global-local enrichments for nonlinear fracture analysis. In: Mattos HdC, Alves M (eds) International Symposium on Mechanics of Solids-MECSOL 2009, ABCM - Brazilian Society of Mechanical Sciences and Engineering, Rio de Janeiro, RJ, Brazil, pp 317-330, iSBN 978-85-85769-43-7 
[30] Kim DJ, Pereira J, Duarte C (2010) Analysis of three-dimensional fracture mechanics problems: A two-scale approach using coarse generalized FEM meshes. International Journal for Numerical Methods in Engineering 81(3):335-365, DOI 10.1002/nme.2690

[31] Kim DJ, Duarte C, Sobh N (2011) Parallel simulations of three-dimensional cracks using the generalized finite element method. Computational Mechanics 47(3):265-282, DOI 10.1007/ s00466-010-0546-5

[32] Lee SH, Song JH, Yoon YC, Zi G, Belytschko T (2004) Combined extended and superimposed finite element method for cracks. International Journal for Numerical Methods in Engineering 59(11191136), dOI: $10.1002 / n m e .908$

[33] Loehnert S, Belytschko T (2007) A multiscale projection method for macro/microcrack simulations. International Journal for Numerical Methods in Engineering 71(12):1466-1482

[34] Melenk J, Babuška I (1996) The partition of unity finite element method: Basic theory and applications. Computer Methods in Applied Mechanics and Engineering 139:289-314

[35] Menk A, Bordas P (2010) Numerically determined enrichment functions for the extended finite element method and applications to bi-material anisotropic fracture and polycrystals. International Journal for Numerical Methods in Engineering 83(7):805828

[36] Mi Y, Aliabadi M (1994) Three-dimensional crack growth simulation using BEM. Computers and Structures 52:871-878

[37] Moës N, Dolbow J, Belytschko T (1999) A finite element method for crack growth without remeshing. International Journal for Numerical Methods in Engineering 46:131-150

[38] Moës N, Gravouil A, Belytschko T (2002) Non-planar 3D crack growth by the extended finite element and level sets - Part I: Mechanical model. International Journal for Numerical Methods in Engineering 53(11):2549-2568

[39] Mousavi S, Grinspun E, Sukumar N (2011) Harmonic enrichment functions: A unified treatment of multiple, intersecting and branched cracks in the extended finite element method. International Journal for Numerical Methods in Engineering 85:13061322, DOI 10.1002/nme.3020

[40] Oden J, Duarte C, Zienkiewicz O (1998) A new cloud-based $h p$ finite element method. Computer Methods in Applied Mechanics and Engineering 153:117-126

[41] O'Hara P, Duarte C, Eason T (2009) Generalized finite element analysis of three-dimensional heat transfer problems exhibiting sharp thermal gradients. Computer Methods in Applied Mechanics and Engineering 198(21-26):1857-1871, DOI 10.1016/j.cma.2008.12.024

[42] O'Hara P, Duarte C, Eason T (2011) Transient analysis of sharp thermal gradients using coarse finite element meshes. Computer Methods in Applied Mechanics and Engineering 200(5-8):812-829, DOI 10.1016/j.cma.2010.10.005

[43] Oskay C, Fish J (2008) On calibration and validation of eigendeformation-based multiscale models for failure analysis of heterogeneous systems. Computational Mechanics 42(2):181-195

[44] Paris A, Erdogan F (1963) A critical analysis of crack propagation laws. Journal of Basic Engineering 85:528-534 
[45] Passieux J, Gravouil A, Rethore J, Baietto M (2010) Direct estimation of generalized stress intensity factors using a three-scale concurrent multigrid X-FEM. International Journal for Numerical Methods in Engineering DOI 10.1002/nme.3037

[46] Pereira J, Duarte C, Guoy D, Jiao X (2009) Hp-Generalized FEM and crack surface representation for non-planar 3-D cracks. International Journal for Numerical Methods in Engineering 77(5):601-633, DOI 10.1002/nme.2419

[47] Pereira J, Duarte C, Jiao X, Guoy D (2009) Generalized finite element method enrichment functions for curved singularities in 3D fracture mechanics problems. Computational Mechanics 44(1):73-92, DOI 10.1007/s00466-008-0356-1

[48] Pereira J, Duarte C, Jiao X (2010) Three-dimensional crack growth with $h p$-generalized finite element and face offsetting methods. Computational Mechanics 46(3):431-453, DOI 10.1007/ s00466-010-0491-3

[49] Pierres E, Baietto M, Gravouil A (2010) A two-scale extended finite element method for modelling 3D crack growth with interfacial contact. Computer Methods in Applied Mechanics and Engineering 199:1165-1177, doi:10.1016/j.cma.2009.12.006

[50] Rannou J, Gravouil A, Baietto-Dubourg M (2009) A local multigrid X-FEM strategy for 3-D crack propagation. International Journal for Numerical Methods in Engineering 77:581-600, doi: 10.1002/nme.2427

[51] Rashid M (1998) The arbitrary local mesh replacement method: An alternative to remeshing for crack propagation analysis. Computer Methods in Applied Mechanics and Engineering 154:133-150

[52] Richard H, Fulland M, Sander M (2005) Theoretical crack path prediction. Fatigue \& Fracture of Engineering Materials \& Structures 28:3-12, http://dx.doi.org/10.1111/j.1460-2695.2004.00855.x

[53] Schöllmann M, Richard H, Kullmer G, Fulland M (2002) A new criterion for the prediction of crack development in multiaxially loaded structures. International Journal of Fracture 117:129-141

[54] Strouboulis T, Copps K, Babuška I (2001) The generalized finite element method. Computer Methods in Applied Mechanics and Engineering 190:4081-4193

[55] Strouboulis T, Zhang L, Babuška I (2003) Generalized finite element method using mesh-based handbooks: Application to problems in domains with many voids. Computer Methods in Applied Mechanics and Engineering 192:3109-3161

[56] Strouboulis T, Zhang L, Babuška I (2004) $p$-version of the generalized FEM using mesh-based handbooks with applications to multiscale problems. International Journal for Numerical Methods in Engineering 60:1639-1672

[57] Sukumar N, Chopp D, Moran B (2003) Extended finite element method and fast marching method for three-dimensional fatigue crack propagation. Engineering Fracture Mechanics 70:29-48

[58] Sukumar N, Chopp D, Béchet E, Moës N (2008) Three-dimensional non-planar crack growth by a coupled extended finite element and fast marching method. International Journal for Numerical Methods in Engineering 76:727-748

[59] Tada H, Paris P, Irwin G (2000) The Stress Analysis of Cracks Handbook, 3rd edn. ASME Press, New York 
[60] Ural A, Heber G, Wawrzynek P, Ingraffea A, Lewicki D, Neto J (2005) Three-dimensional, parallel, finite element simulation of fatigue crack growth in a spiral bevel pinion gear. Engineering Fracture Mechanics 72:1148-1170 